Pacific

Journal of

Mathematics

COBOUNDARY LIE BIALGEBRAS AND COMMUTATIVE SUBALGEBRAS OF UNIVERSAL ENVELOPING ALGEBRAS

BenJamin EnRIQUeZ and Gilles Halbout

Volume 229 No. 1

January 2007 


\title{
COBOUNDARY LIE BIALGEBRAS AND COMMUTATIVE SUBALGEBRAS OF UNIVERSAL ENVELOPING ALGEBRAS
}

\author{
BENJAMIN ENRIQUEZ AND Gilles Halbout
}

\begin{abstract}
We solve a functional version of the problem of twist quantization of a coboundary Lie bialgebra $(\mathfrak{g}, r, Z)$. We derive from this that the formal Poisson manifolds $\mathfrak{g}^{*}$ and $G^{*}$ are isomorphic, and we construct an injective algebra morphism $S\left(\mathfrak{g}^{*}\right)^{\mathfrak{g}} \hookrightarrow U\left(\mathfrak{g}^{*}\right)$. When $(\mathfrak{g}, r, Z)$ can be quantized, we construct a deformation of this morphism. In the particular case when $\mathfrak{g}$ is quasitriangular and nondegenerate, we compare our construction with Semenov-Tian-Shansky's construction of a commutative subalgebra of $U\left(\mathfrak{g}^{*}\right)$. We also show that the canonical derivation of the function ring of $G^{*}$ is Hamiltonian.
\end{abstract}

\section{Introduction}

Let $(\mathfrak{g}, r, Z)$ be a coboundary Lie bialgebra over a field $\mathbb{K}$ of characteristic 0 . This means that $\mathfrak{g}$ is a Lie bialgebra, the Lie cobracket $\delta$ of which is the coboundary of $r \in \bigwedge^{2}(\mathfrak{g})$ : in symbols, $\delta(x)=[x \otimes 1+1 \otimes x, r]$ for any $x \in \mathfrak{g}$. This condition means that $Z:=\operatorname{CYB}(r)$ belongs to $\wedge^{3}(\mathfrak{g})^{\mathfrak{g}}$ (here CYB is the left hand side of the classical Yang-Baxter equation). Quasitriangular and triangular Lie bialgebras are particular cases of this definition.

It is an open question to construct a twist quantization of $(\mathfrak{g}, r, Z)$, that is, a pair $(J, \Phi)$, where $J \in U(\mathfrak{g})^{\otimes 2} \llbracket \hbar \rrbracket$ and $\Phi \in U(\mathfrak{g})^{\otimes 3} \llbracket \hbar \rrbracket$ are invertible $(\hbar$ is a formal series), $\Phi$ is $\mathfrak{g}$-invariant, $(J, \Phi)$ satisfies a cocycle relation and deforms $(r, Z)$. If $(J, \Phi)$ satisfies these conditions, then $\Phi$ satisfies the pentagon relation, is $\mathfrak{g}$-invariant and deforms $Z$. Such a $\Phi$ is called a quantization of $Z$. Drinfeld [1989b, Proposition 3.10] constructed a quantization $\Phi$ of $Z$. Any pair $(J, \Phi)$ can be made admissible (in the sense of [Enriquez and Halbout 2004]), and the associated formal functions $(\rho, \varphi)$ then satisfy functional analogues of the pentagon and cocycle equations (this is explained in Section 6). We call this system of equations the functional analogue of twist quantization.

We describe the set of solutions of the functional analogue of twist quantization for $(\mathfrak{g}, r, Z)$. Namely, we derive from Drinfeld's result that $Z$ can be lifted to an

MSC2000: primary 17B63; secondary 17B80, 16W30.

Keywords: coboundary Lie bialgebras, commuting families, linearization, Poisson algebras. 
element $\varphi \in \mathfrak{m}_{\mathrm{g}^{*}}^{\widehat{\otimes} 3}$ satisfying the functional pentagon relation (Proposition 2.1). We then prove that $r$ can be lifted to an element $\rho \in \mathfrak{m}_{\mathfrak{g}^{*}}^{\widehat{\otimes} 2}$, such that $(\rho, \varphi)$ satisfies the functional cocycle relation (Theorem 2.3); here $\mathfrak{m}_{\mathfrak{g}^{*}} \subset \mathbb{O}_{\mathfrak{g}^{*}}$ is the maximal ideal of the ring of formal functions on $\mathfrak{g}^{*}$. We show that all solutions are related by suitable gauge transformations.

The first corollary is that the formal Poisson manifolds $\mathfrak{g}^{*}$ and $G^{*}$ are isomorphic (Corollary 3.1).

In Section 4, we prove another corollary (Theorem 4.3): we construct an injection of algebras $S\left(\mathfrak{g}^{*}\right)^{\mathfrak{g}} \hookrightarrow U\left(\mathfrak{g}^{*}\right)$ (here $\mathfrak{g}$ acts on $S\left(\mathfrak{g}^{*}\right)$ by symmetric powers of the coadjoint action). This morphism is filtered; its associated graded morphism is the canonical inclusion $S\left(\mathfrak{g}^{*}\right)^{\mathfrak{g}} \subset S\left(\mathfrak{g}^{*}\right)$. This way, we obtain a commutative subalgebra of $U\left(\mathfrak{g}^{*}\right)$. The fact that the graded subalgebra $S\left(\mathfrak{g}^{*}\right)^{\mathfrak{g}} \subset S\left(\mathfrak{g}^{*}\right)$ is Poisson commutative can be viewed as a classical limit of this situation. It can either be viewed as a corollary of the fact that $\mathcal{O}_{G}^{\mathfrak{g}} \subset \mathrm{O}_{G}$ is a Poisson commutative subalgebra, or it can be proved directly (Lemma 4.2); here $O_{G}$ is the ring of formal functions on $G$, on which $\mathfrak{g}$ acts by conjugation.

In Sections 7 and 8, assuming the existence of a twist quantization of $\mathfrak{g}$, we construct formal deformations of the algebra inclusions $S\left(\mathfrak{g}^{*}\right)^{\mathfrak{g}} \hookrightarrow U\left(\mathfrak{g}^{*}\right)$ and $\mathcal{O}_{G}^{\mathfrak{g}} \subset \mathcal{O}_{G^{*}}$. All these results use the theory of duality of QUE (quantized universal enveloping) and QFSH (quantized formal series Hopf) algebras; this theory is recalled in Section 5.

In Section 9, we assume that $\mathfrak{g}$ is quasitriangular. In that case, we show that $U\left(\mathfrak{g}^{*}\right)$ contains a family $C_{s}$ of commutative subalgebras, indexed by $s \in \mathbb{K}$; this result may be viewed as a classical limit of Drinfeld's result about commutativity of twisted traces. We explain why only $C_{0}$ has an analogue in the general coboundary case. Semenov-Tian-Shansky [1984] defined an algebra morphism $U(\mathfrak{g}) \stackrel{\mathfrak{g}}{\text { STS }} U\left(\mathfrak{g}^{*}\right)$; we show that its image coincides with $C_{1}$, and is therefore in general different from the image $C_{0}$ of the morphism in our construction.

Finally, in Section 10, we show that the canonical derivation of $0_{G^{*}}$ is Hamiltonian. This derivation is equal to $\hbar^{-1}\left(S^{2}-\mathrm{id}\right)_{\mid \hbar=0}$, where $S$ is the antipode of any quantization of $\mathfrak{O}_{G^{*}}$.

Notation. We use the standard notation for the coproduct-insertion maps: we say that an ordered set is a pair of a finite set $S$ and a bijection $\{1, \ldots,|S|\} \rightarrow S$. Given $I_{1}, \ldots, I_{m}$ disjoint ordered subsets of $\{1, \ldots, n\}$, a Hopf algebra $(U, \Delta)$, and $a \in U^{\otimes m}$, we define

$$
a^{I_{1}, \ldots, I_{n}}=\sigma_{I_{1}, \ldots, I_{m}} \circ\left(\Delta^{\left|I_{1}\right|} \otimes \cdots \otimes \Delta^{\left|I_{n}\right|}\right)(a),
$$

with $\Delta^{(1)}=\mathrm{id}, \Delta^{(2)}=\Delta, \Delta^{(n+1)}=\left(\mathrm{id}^{\otimes n-1} \otimes \Delta\right) \circ \Delta^{(n)}$, and $\sigma_{I_{1}, \ldots, I_{m}}: U^{\otimes \sum_{i}\left|I_{i}\right|} \rightarrow$ $U^{\otimes n}$ is the morphism corresponding to the map $\left\{1, \ldots, \sum_{i}\left|I_{i}\right|\right\} \rightarrow\{1, \ldots, n\}$ 
taking $\left(1, \ldots,\left|I_{1}\right|\right)$ to $I_{1},\left(\left|I_{1}\right|+1, \ldots,\left|I_{1}\right|+\left|I_{2}\right|\right)$ to $I_{2}$, etc. When $U$ is cocommutative, this definition depends only on the sets underlying $I_{1}, \ldots, I_{m}$.

\section{Solutions of the functional twist equations}

If $\mathfrak{g}$ is a Lie algebra, we denote by $\widehat{O}_{\mathfrak{g}^{*}}=\widehat{S}(\mathfrak{g})$ the formal series ring of functions on the formal neighborhood of 0 in $\mathfrak{g}^{*}$. We define by $\mathfrak{m}_{\mathfrak{g}^{*}} \subset \mathcal{O}_{\mathfrak{g}^{*}}$ the maximal ideal of this ring. If $k$ is an integer $\geq 1$, we denote by $O_{\left(\mathfrak{g}^{*}\right)^{k}}=\widehat{S}(\mathfrak{g})^{\widehat{\otimes} k}$ the ring of formal functions on $\left(\mathfrak{g}^{*}\right)^{k}$, by $\mathfrak{m}_{\left(\mathfrak{g}^{*}\right)^{k}}$ its maximal ideal, and by $\mathfrak{m}_{\left(\mathfrak{g}^{*}\right)^{k}}^{i}$ the $i$-th power of this ideal. Here, $\widehat{\otimes}$ is the completed tensor product, defined by $V_{0} \llbracket x_{1}, \ldots, x_{n} \rrbracket \widehat{\otimes} W_{0} \llbracket y_{1}, \ldots, y_{n} \rrbracket:=V_{0} \otimes W_{0} \llbracket x_{1}, \ldots, y_{n} \rrbracket$, where $V_{0}, W_{0}$ are vector spaces.

When equipped with the Poisson bracket, $\mathfrak{m}_{\left(\mathfrak{g}^{*}\right)^{k}}^{2}$ is a pronilpotent Lie algebra. If $\mathfrak{a}$ is such a Lie algebra, the Campbell-Baker-Hausdorff series $x \star y=x+y+$ $\frac{1}{2}[x, y]+\cdots+B_{n}(x, y)+\cdots$ converges and defines a group structure on $\mathfrak{a}$. In particular, if $f, g \in \mathfrak{m}_{\left(\mathfrak{g}^{*}\right)^{k}}^{2}$, the product $f \star g=f+g+\frac{1}{2}\{f, g\}+\cdots+B_{n}(f, g)+\cdots$ is convergent and defines a group structure on $\mathfrak{m}_{\left(\mathfrak{g}^{*}\right)^{k}}^{2}$.

If $f \in \mathcal{O}_{\mathfrak{g}^{*}}^{\widehat{\otimes} n}$ and $P_{1}, \ldots, P_{m}$ are disjoint subsets of $\{1, \ldots, m\}$, one defines $f^{P_{1}, \ldots, P_{n}}$ as in the Introduction using the cocommutative coproduct $\Delta_{0}$ of $\mathscr{O}_{\mathfrak{g}^{*}}$ (dual to the addition of $\mathfrak{g}^{*}$ ).

Let $\mathfrak{g}$ be a Lie algebra and $Z \in \bigwedge^{3}(\mathfrak{g})^{\mathfrak{g}}$.

Proposition 2.1. There exists $\varphi \in\left(\mathfrak{m}_{\mathfrak{g}^{*}}^{\widehat{\otimes} 3}\right)^{\mathfrak{g}}\left(\subset \mathfrak{m}_{\left(\mathfrak{g}^{*}\right)^{3}}^{2}\right)$ satisfying the functional pentagon equation

$$
\varphi^{1,2,34} \star \varphi^{12,3,4}=\varphi^{2,3,4} \star \varphi^{1,23,4} \star \varphi^{1,2,3},
$$

whose image under the map $\mathfrak{m}_{\mathfrak{g}^{*}}^{\widehat{\otimes} 3} \rightarrow\left(\mathfrak{m}_{\mathfrak{g}^{*}} / \mathfrak{m}_{\mathfrak{g}^{*}}^{2}\right)^{\otimes 3}=\mathfrak{g}^{\otimes 3} \stackrel{\text { Alt }}{\rightarrow} \bigwedge^{3}(\mathfrak{g})$ equals $Z$ (here Alt is the total antisymmetrization map). Such a $\varphi$ (we call it a lift of $Z$ ) is unique up to the action of an element of $\left(\mathfrak{m}_{\mathfrak{g}^{*}}^{\widehat{\widehat{\alpha}}}\right)^{\mathfrak{g}}$ by $\sigma \cdot \varphi=\sigma^{2,3} \star \sigma^{1,23} \star \varphi \star(-\sigma)^{12,3} \star(-\sigma)^{1,2}$.

Proof. Drinfeld [1989b, Proposition 3.10] constructed a solution $\Phi \in U(\mathfrak{g})^{\otimes 3} \llbracket \hbar \rrbracket$ of the pentagon equation

$$
\Phi^{1,2,34} \Phi^{12,3,4}=\Phi^{2,3,4} \Phi^{1,23,4} \Phi^{1,2,3}
$$

such that $\varepsilon^{(2)}(\Phi)=1$ and $\Phi=1^{\otimes 3}+O(\hbar)$ (here $\varepsilon^{(2)}=\mathrm{id} \otimes \varepsilon \otimes \mathrm{id}$; applying $\varepsilon$ to the second and third factors of $(2-1)$, we also get $\left.\varepsilon^{(1)}(\Phi)=\varepsilon^{(3)}(\Phi)=1\right)$.

In [Enriquez and Halbout 2004], we stated that $\Phi$ can be transformed into an admissible solution $\Phi^{\prime}$ of the same equations, using an invariant twist. In the Appendix, we explain why the proof given in [Enriquez and Halbout 2004] is wrong, and we give a correct proof. The condition that $\Phi^{\prime}$ is admissible means that it belongs to $\left(U(\mathfrak{g}) \llbracket \hbar \rrbracket^{\prime}\right)^{\widehat{\otimes} 3}$, where $U(\mathfrak{g}) \llbracket \hbar \rrbracket^{\prime}=U(\hbar \mathfrak{g} \llbracket \hbar \rrbracket) \subset U(\mathfrak{g}) \llbracket \hbar \rrbracket$ is the 
subalgebra generated by $\mathfrak{g} \llbracket \hbar \rrbracket$. Then $U(\mathfrak{g}) \llbracket \hbar \rrbracket^{\prime}$ is a formal deformation of the Poisson algebra $\widehat{S}(\mathfrak{g})$.

Then the reduction mod $\hbar$ of $\hbar \log \Phi^{\prime}$ belongs to $\widehat{S}(\mathfrak{g})^{\widehat{\otimes} 3}$, and in fact to $\mathfrak{m}_{\mathfrak{g}^{*}}^{\widehat{\widehat{3}}}$. Since $\Phi^{\prime}$ satisfies (2-1), this reduction satisfies the functional pentagon equation. This gives the existence of $\varphi$. One can also construct $\varphi$ directly using cohomological methods, as will be done for $\rho$ later.

To prove uniqueness, let $\varphi$ and $\varphi^{\prime}$ be lifts of $Z$. The classes of $\varphi$ and $\varphi^{\prime}$ are the same in $\mathfrak{m}_{\mathfrak{g}^{*}}^{\widehat{\otimes} 3} /\left(\mathfrak{m}_{\mathfrak{g}^{*}}^{\widehat{\otimes} 3} \cap \mathfrak{m}_{\left(\mathfrak{g}^{*}\right)^{3}}^{3}\right)$, as this space is 0 . Let $N$ be an integer $\geq 3$; assume that we have found $\sigma_{N} \in\left(\mathfrak{m}_{\mathfrak{g}^{*}}^{\widehat{\otimes} 2}\right)^{\mathfrak{g}}$ such that $\sigma_{N} \cdot \varphi$ and $\varphi^{\prime}$ are equal modulo $\mathfrak{m}_{\mathfrak{g}^{*}}^{\widehat{\otimes} 3} \cap \mathfrak{m}_{\left(\mathfrak{g}^{*}\right)^{3}}^{N}$. Write $\varphi^{\prime}=\sigma_{N} \cdot \varphi+\psi$, with $\psi \in\left(\mathfrak{m}_{\mathfrak{g}^{*}}^{\widehat{\otimes} 3} \cap \mathfrak{m}_{\left(\mathfrak{g}^{*}\right)^{3}}^{N}\right)^{\mathfrak{g}}$.

Lemma 2.2 [Enriquez et al. 2003, p. 2477]. For any $k \geq 1, n \geq 2, f, h \in \mathfrak{m}_{\left(\mathfrak{g}^{*}\right)^{k}}^{2}$ and $g \in \mathfrak{m}_{\left(\mathfrak{g}^{*}\right)^{k}}^{n}$, one has

$$
f \star(h+g)=f \star h+g, \quad(f+g) \star h=f \star h+g \text { modulo } \mathfrak{m}_{\left(\mathfrak{g}^{*}\right)^{k}}^{n+1} .
$$
Then

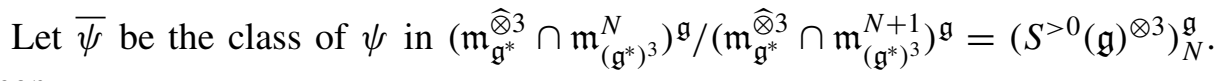

$$
\bar{\psi}^{1,2,34}+\bar{\psi}^{12,3,4}=\bar{\psi}^{2,3,4}+\bar{\psi}^{1,23,4}+\bar{\psi}^{1,2,3},
$$

which means that $\bar{\psi}$ is a cocycle in the subcomplex $\left(\left(S^{>0}(\mathfrak{g})^{\otimes \cdot}\right)^{\mathfrak{g}}, d\right)$ of the $\operatorname{Cartier}^{1}$ complex $\left(S(\mathfrak{g})^{\otimes \cdot}, d\right)$. Using [Drinfeld 1989b, Proposition 3.11], one can prove that the $k$-th cohomology group of this complex is $\Lambda^{k}(\mathfrak{g})^{\mathfrak{g}}$ and that the antisymmetrization map coincides with the canonical map from the space of cocycles to the cohomology. For $N=3$, the hypothesis implies that $\operatorname{Alt}(\bar{\psi})=0$, so $\bar{\psi}$ is a coboundary of an element $\bar{\tau}_{3} \in\left(S^{>0}(\mathfrak{g})^{\otimes 2}\right)_{3}^{\mathfrak{g}}$. For $N>3, \bar{\psi}$ is the coboundary of an element $\bar{\tau}_{N} \in\left(S^{>0}(\mathfrak{g})^{\otimes 2}\right)_{N}^{\mathfrak{g}}$, since the degree $N$ part of the relevant cohomology group vanishes. We then set $\sigma_{N+1}=\sigma_{N}+\tau_{N}$, where

$$
\tau_{N} \in\left(\mathfrak{m}_{\mathfrak{g}^{*}}^{\widehat{\otimes} 2} \cap \mathfrak{m}_{\left(\mathfrak{g}^{*}\right)^{2}}^{N} \mathfrak{g}\right.
$$

is a lift of $\bar{\tau}_{N}$. Then $\sigma_{N+1} \cdot \varphi$ and $\varphi^{\prime}$ are equal modulo $\mathfrak{m}_{\mathfrak{g}^{*}}^{\widehat{\widehat{Q}} 3} \cap \mathfrak{m}_{\left(\mathfrak{g}^{*}\right)^{3}}^{N+1}$. The sequence $\left(\sigma_{N}\right)_{N \geq 3}$ has a limit $\sigma$. Then $\sigma \cdot \varphi=\varphi^{\prime}$.

We now construct a lift of $r$ :

Theorem 2.3. There exists $\rho \in \mathfrak{m}_{\mathfrak{g}^{*}}^{\widehat{\otimes} 2}$ such that

$$
\rho^{1,2} \star \rho^{12,3}=\rho^{2,3} \star \rho^{1,23} \star \varphi,
$$

and whose image in $\mathfrak{g}^{\otimes 2}$ under the square of the projection $\mathfrak{m}_{\mathfrak{g}^{*}} \rightarrow \mathfrak{m}_{\mathfrak{g}^{*}} / \mathfrak{m}_{\mathfrak{g}^{*}}^{2}=\mathfrak{g}$ equals $r$. Such a $\rho$ (we call it a lift of $r$ ) is unique up to the action of $\mathfrak{m}_{\mathfrak{g}^{*}}$ by $\lambda \cdot \rho=\lambda^{1} \star \lambda^{2} \star \rho \star(-\lambda)^{12}$. We call Equation (2-2) the functional cocycle equation.

\footnotetext{
${ }^{1}$ We denote by $S(\mathfrak{g})$ the symmetric algebra of $\mathfrak{g}$, and by $S^{>0}(\mathfrak{g})$ the positive degree part; the index $N$ means the part of total degree $N$.
} 
Proof. We construct $\rho$ by induction: we will construct a convergent sequence

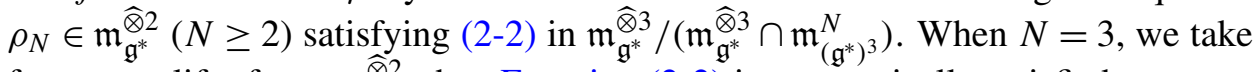
for $\rho_{2}$ any lift of $r$ to $\mathfrak{m}_{\mathfrak{g}^{*}}^{\widehat{\otimes}}$; then Equation (2-2) is automatically satisfied.

Let $N$ be an integer $\geq 3$; assume that we have constructed $\rho_{N}$ in $\mathfrak{m}_{\mathfrak{g}^{*}}^{\widehat{\otimes} 2}$ satisfying Equation (2-2) in $\mathfrak{m}_{\mathfrak{g}^{*}}^{\widehat{\otimes} 3} /\left(\mathfrak{m}_{\mathfrak{g}^{*}}^{\widehat{\otimes} 3} \cap \mathfrak{m}_{\left(\mathfrak{g}^{*}\right)^{3}}^{N}\right)$. Set

$$
\alpha_{N}:=\rho_{N}^{1,2} \star \rho_{N}^{12,3}-\rho_{N}^{2,3} \star \rho_{N}^{1,23} \star \varphi .
$$

Then $\alpha_{N}$ belongs to

$$
\mathfrak{m}_{\mathfrak{g}^{*}}^{\widehat{\otimes} 3} \cap \mathfrak{m}_{\left(\mathfrak{g}^{*}\right)^{3}}^{N},
$$

and the following equalities hold in $\mathfrak{m}_{\mathfrak{g}^{*}}^{\widehat{\otimes} 4} /\left(\mathfrak{m}_{\mathfrak{g}^{*}}^{\widehat{\otimes} 4} \cap \mathfrak{m}_{\left(\mathfrak{g}^{*}\right)^{4}}^{N+1}\right)$ :

$$
\begin{aligned}
\alpha_{N}^{12,3,4}= & \rho_{N}^{1,2} \star \alpha_{N}^{12,3,4}=\rho_{N}^{1,2} \star \rho_{N}^{12,3} \star \rho_{N}^{123,4}-\rho_{N}^{1,2} \star \rho_{N}^{3,4} \star \rho_{N}^{12,34} \star \varphi^{12,3,4} \\
& (\text { using Lemma 2.2) } \\
= & \left(\alpha_{N}^{1,2,3}+\rho_{N}^{2,3} \star \rho_{N}^{1,23} \star \varphi^{1,2,3}\right) \star \rho_{N}^{123,4}-\rho_{N}^{3,4} \star \rho_{N}^{1,2} \star \rho_{N}^{12,34} \star \varphi^{12,3,4} \\
= & \alpha_{N}^{1,2,3}+\rho_{N}^{2,3} \star \rho_{N}^{1,23} \star \rho_{N}^{123,4} \star \varphi^{1,2,3}-\rho_{N}^{3,4} \star\left(\rho_{N}^{2,34} \star \rho_{N}^{1,234} \star \varphi^{1,2,34}\right. \\
& \left.+\alpha_{N}^{1,2,34}\right) \star \varphi^{12,3,4}
\end{aligned}
$$

(using Lemma 2.2, the invariance of $\varphi$ and the definition of $\alpha_{N}^{1,2,34}$ )

$$
\begin{aligned}
= & \alpha_{N}^{1,2,3}+\rho_{N}^{2,3} \star\left(\alpha_{N}^{1,23,4}+\rho_{N}^{23,4} \star \rho_{N}^{1,234} \star \varphi^{1,23,4}\right) \star \varphi^{1,2,3} \\
& -\alpha_{N}^{1,2,34}-\rho_{N}^{3,4} \star \rho_{N}^{2,34} \star \rho_{N}^{1,234} \star \varphi^{1,2,34} \star \varphi^{12,3,4}
\end{aligned}
$$

(using the definition of $\alpha_{N}^{1,23,4}$ and Lemma 2.2)

$$
\begin{aligned}
= & \alpha_{N}^{1,2,3}+\alpha_{N}^{1,23,4}+\left(\rho_{N}^{3,4} \star \rho_{N}^{2,34} \star \varphi^{2,3,4}+\alpha_{N}^{2,3,4}\right) \star \rho_{N}^{1,234} \star \varphi^{1,23,4} \star \varphi^{1,2,3} \\
& -\alpha_{N}^{1,2,34}-\rho_{N}^{3,4} \star \rho_{N}^{2,34} \star \rho_{N}^{1,234} \star \varphi^{1,2,34} \star \varphi^{12,3,4}
\end{aligned}
$$

(using the definition of $\alpha_{N}^{2,3,4}$ and Lemma 2.2)

$$
=\alpha_{N}^{1,2,3}+\alpha_{N}^{1,23,4}-\alpha_{N}^{1,2,34}+\alpha_{N}^{2,3,4}
$$

(using Lemma 2.2, the invariance of $\varphi$ and the fact that $\varphi$ satisfies the functional pentagon equation). Then

Denote by $\bar{\alpha}_{N}$ the image of $\alpha_{N}$ in $\left(\mathfrak{m}_{\mathfrak{g}^{*}}^{\widehat{\otimes}} \cap \mathfrak{m}_{\left(\mathfrak{g}^{*}\right)^{3}}^{N}\right) /\left(\mathfrak{m}_{\mathfrak{g}^{*}}^{\widehat{\otimes} 3} \cap \mathfrak{m}_{\left(\mathfrak{g}^{*}\right)^{3}}^{N+1}\right)=\left(S^{>0}(\mathfrak{g})^{\otimes 3}\right)_{N}$.

$$
\bar{\alpha}_{N}^{12,3,4}+\bar{\alpha}_{N}^{1,2,34}=\bar{\alpha}_{N}^{1,2,3}+\bar{\alpha}_{N}^{1,23,4}+\bar{\alpha}_{N}^{2,3,4} .
$$

Thus $\bar{\alpha}_{N}$ is a cocycle for the subcomplex $\left(S^{>0}(\mathfrak{g})^{\otimes}, d\right)$ of the Cartier complex. Using [Drinfeld 1989b, Proposition 3.11], one proves that the $k$-th cohomology group of this subcomplex is $\Lambda^{k}(\mathfrak{g})$, and that antisymmetrization coincides with the canonical projection from the space of cocycles to the cohomology group. For $N=3$, the equation $\operatorname{CYB}(r)=Z$ implies $\operatorname{Alt}\left(\bar{\alpha}_{3}\right)=0$, hence $\bar{\alpha}_{3}$ is the coboundary 
of an element $\bar{\beta}_{3} \in\left(S^{>0}(\mathfrak{g})^{\otimes 2}\right)_{3}$. For $N>3, \bar{\alpha}_{N}$ is the coboundary of an element $\bar{\beta}_{N} \in\left(S^{>0}(\mathfrak{g})^{\otimes 2}\right)_{N}$, since the degree $N$ part of the cohomology vanishes. We set $\rho_{N+1}:=\rho_{N}+\beta_{N}$, where $\beta_{N} \in \mathfrak{m}_{\mathfrak{g}^{*}}^{\widehat{\widehat{ }} 2} \cap \mathfrak{m}_{\left(\mathfrak{g}^{*}\right)^{2}}^{N}$ is a representative of $\bar{\beta}_{N}$. Then $\rho_{N+1}$ satisfies (2-2) in

$$
\mathfrak{m}_{\mathfrak{g}^{*}}^{\widehat{\otimes} 3} /\left(\mathfrak{m}_{\mathfrak{g}^{*}}^{\widehat{\otimes} 3} \cap \mathfrak{m}_{\left(\mathfrak{g}^{*}\right)^{3}}^{N+1}\right)
$$

The sequence $\left(\rho_{N}\right)_{N \geq 2}$ has a limit $\rho$, which satisfies (2-2).

The second part of the theorem can be proved either by analyzing the choices for $\bar{\beta}_{N}$ in the proof above, or by following the proof of the previous proposition.

Remark 2.4. If $\varphi$ is replaced by $\varphi^{\prime}=\sigma \star \varphi$, then a solution of (2-2) is $\rho^{\prime}=\rho \star(-\sigma)$.

\section{Isomorphism of formal Poisson manifolds $\mathfrak{g}^{*} \simeq G^{*}$}

We assume that $\mathfrak{g}$ is a finite dimensional coboundary Lie bialgebra. The following result was proved in [Enriquez et al. 2005] when $\mathfrak{g}$ is quasitriangular; that result is itself a generalization of the formal version of the Ginzburg-Weinstein isomorphism [Ginzburg and Weinstein 1992; Alekseev 1997; Boalch 2001]. On the other hand, one knows that linearization does not hold for general Poisson-Lie groups [Chloup-Arnould 1997].

Corollary 3.1. There exists an isomorphism of formal Poisson manifolds $\mathfrak{g}^{*} \simeq G^{*}$. Proof. Let $P: \wedge^{2}\left(\mathcal{O}_{\mathfrak{g}^{*}}\right) \rightarrow \mathcal{O}_{\mathfrak{g}^{*}}$ be the Poisson bracket on $\mathcal{O}_{\mathfrak{g}^{*}}$ corresponding to the Lie-Poisson (or Kostant-Kirillov-Souriau, or linear Poisson) structure on $\mathfrak{g}^{*}$. Let $m_{0}$ be the product and $\Delta_{0}$ be the cocommutative coproduct of $\mathcal{O}_{\mathfrak{g}^{*}} \simeq \widehat{S}(\mathfrak{g})$ (recall that elements of $\mathfrak{g}$ are primitive for $\left.\Delta_{0}\right)$. Then $\left(\mathcal{O}_{\mathfrak{g}^{*}}, m_{0}, P, \Delta_{0}\right)$ is a Poisson formal series Hopf (PFSH) algebra ${ }^{2}$; it corresponds to the formal Poisson-Lie group $\left(\mathfrak{g}^{*},+\right)$ equipped with its Lie-Poisson structure.

Set ${ }^{\rho} \Delta_{0}(f)=\rho \star \Delta_{0}(f) \star(-\rho)$ for any $f \in \mathcal{O}_{\mathfrak{g}^{*}}$. It follows from the fact that $\rho$ satisfies the functional cocycle equation Equation (2-2) that $\left(\mathcal{O}_{\mathfrak{g}^{*}}, m_{0}, P,{ }^{\rho} \Delta_{0}\right)$ is a PFSH algebra.

Denote by PFSHA and LBA the categories of PSFH algebras and Lie bialgebras. We have a category equivalence $c:$ PFSHA $\rightarrow$ LBA, taking $(\mathcal{O}, m, P, \Delta)$ to the Lie bialgebra $(\mathfrak{c}, \mu, \delta)$, where $\mathfrak{c}:=\mathfrak{m} / \mathfrak{m}^{2}(\mathfrak{m} \subset \mathcal{O}$ is the maximal ideal), the Lie cobracket of $\mathfrak{c}$ is induced by $\Delta-\Delta^{2,1}: \mathfrak{m} \rightarrow \bigwedge^{2}(\mathfrak{m})$, and the Lie bracket of $\mathfrak{c}$ is induced by the Poisson bracket $P: \wedge^{2}(\mathfrak{m}) \rightarrow \mathfrak{m}$. The inverse of the functor $c$ takes $(\mathfrak{c}, \mu, \delta)$ to $\mathcal{O}=\widehat{S}(\mathfrak{c})$ equipped with its usual product; $\Delta$ depends only on $\delta$, and $P$ depends on $(\mu, \delta)$.

\footnotetext{
${ }^{2}$ Recall that a PFSH algebra $\left(A, m_{A}, P_{A}, \Delta_{A}\right)$ is a quadruple such that $\left(A, m_{A}\right)$ is a formal series algebra, $\left(A, m_{A}, \Delta_{A}\right)$ is a topological Hopf algebra, $P_{A}$ is a Poisson structure on $A$, and $\Delta_{A}$ : $A \rightarrow A^{\widehat{\otimes} 2}$ is a morphism of Poisson algebras.
} 
Then $c$ restricts to a category equivalence $c_{\mathrm{fd}}$ : $\mathrm{PFSHA}_{\mathrm{fd}} \rightarrow \mathrm{LBA}_{\mathrm{fd}}$ of subcategories of finite-dimensional objects (in the case of PFSH, we say that $\mathrm{O}$ is finitedimensional if and only if $\mathfrak{m} / \mathfrak{m}^{2}$ is).

Let dual: $\mathrm{LBA}_{\mathrm{fd}} \rightarrow \mathrm{LBA}_{\mathrm{fd}}$ be the duality functor. It is a category antiequivalence; we have dual $(\mathfrak{g}, \mu, \delta)=\left(\mathfrak{g}^{*}, \delta^{t}, \mu^{t}\right)$. Then dual $\circ c_{\mathrm{fd}}: \operatorname{PFSHA}_{\mathrm{fd}} \rightarrow \mathrm{LBA}_{\mathrm{fd}}$ is a category antiequivalence. Its inverse is the usual functor $\mathfrak{g} \mapsto U(\mathfrak{g})^{*}$. If $G$ is the formal Poisson-Lie group with Lie bialgebra $\mathfrak{g}$, one sets $\mathscr{O}_{G}=U(\mathfrak{g})^{*}$.

We apply the functor $c$ to $\left(\mathcal{O}_{\mathfrak{g}^{*}}, m_{0}, P,{ }^{\rho} \Delta_{0}\right)$. We obtain $\mathfrak{c}=\mathfrak{m} / \mathfrak{m}^{2}=\mathfrak{g}$; the Lie bracket is unchanged with respect to the case $\rho=0$, so it is the Lie bracket of $\mathfrak{g}$. The Lie cobracket is given by $\delta(x)=[r, x \otimes 1+1 \otimes x]$, since the reduction of $\rho$ modulo $\left(\mathfrak{m}_{\mathfrak{g}^{*}}\right)^{2} \widehat{\otimes} \mathfrak{m}_{\mathfrak{g}^{*}}+\mathfrak{m}_{\mathfrak{g}^{*}} \widehat{\otimes}\left(\mathfrak{m}_{\mathfrak{g}^{*}}\right)^{2}$ is equal to $r$.

Then applying dual $\circ c_{\mathrm{fd}}$ to $\left(\mathrm{O}_{\mathfrak{g}^{*}}, m_{0}, P,{ }^{\rho} \Delta_{0}\right)$, we obtain the Lie bialgebra $\mathfrak{g}^{*}$. So this PFSH algebra is isomorphic to the PFSH algebra of the formal Poisson-Lie group $G^{*}$. In particular, the Poisson algebras $O_{\mathfrak{g}^{*}}$ and $O_{G^{*}}$ are isomorphic. It is easy to check that the map $\mathfrak{g}=\mathfrak{m}_{\mathfrak{g}^{*}} / \mathfrak{m}_{\mathfrak{g}^{*}}^{2} \rightarrow \mathfrak{m}_{G^{*}} / \mathfrak{m}_{G^{*}}^{2}=\mathfrak{g}$ induced by this isomorphism is the identity (here $\mathfrak{m}_{G^{*}} \subset \mathrm{O}_{G^{*}}$ is the maximal ideal).

Remark 3.2. When $\mathfrak{g}$ is infinite dimensional, one can define $\mathscr{O}_{G^{*}}$ as the image of $\mathfrak{g}$ under LBA $\rightarrow$ PFSHA, and then show that the Poisson algebras $\mathscr{O}_{G^{*}}$ and $\mathcal{O}_{\mathfrak{g}^{*}}=(\widehat{S}(\mathfrak{g})$, linear Poisson structure $)$ are isomorphic.

\section{The morphism $S\left(\mathfrak{g}^{*}\right)^{\mathfrak{g}} \hookrightarrow U\left(\mathfrak{g}^{*}\right)$}

In this section, $\mathfrak{g}$ is a finite dimensional coboundary Lie bialgebra.

Lemma 4.1 [Semenov-Tian-Shansky 1985]. $\mathcal{O}_{G}^{\mathfrak{g}} \subset \mathrm{O}_{G}$ is a Poisson commutative subalgebra.

Here the action of $\mathfrak{g}$ on $\mathscr{O}_{G}$ corresponds to the adjoint action of $G$. We recall the proof: if $f, g \in \mathbb{O}_{G}$, then $\{f, g\}=m((\mathbf{L}-\mathbf{R})(r)(f \otimes g))$, where $\mathbf{L}, \mathbf{R}$ are the infinitesimal left and right actions, and $m$ is the product map. If $\varphi \in \mathbb{O}_{G}^{\mathfrak{g}}$, then $\mathbf{L}(a)(\varphi)=\mathbf{R}(a)(\varphi)$ for any $a \in \mathfrak{g}$. Therefore if $f, g \in \mathbb{O}_{G}^{\mathfrak{g}}$, then $(\mathbf{L}-\mathbf{R})(r)(f \otimes g)$ vanishes, hence $\{f, g\}=0$.

The inclusion $\mathcal{O}_{G}^{\mathfrak{g}} \subset \mathscr{O}_{G}$ is a morphism of Poisson algebras with a decreasing filtration. By passing to the associated graded algebras, we obtain:

Lemma 4.2. $S\left(\mathfrak{g}^{*}\right)^{\mathfrak{g}} \subset S\left(\mathfrak{g}^{*}\right)$ is a Poisson commutative subalgebra.

Proof. If $\alpha, \beta \in \mathfrak{g}^{*}$, then $[\alpha, \beta]=\operatorname{ad}^{*}(R(\beta))(\alpha)-\operatorname{ad}^{*}(R(\alpha))(\beta)$, where $R: \mathfrak{g}^{*} \mapsto \mathfrak{g}$ is given by $R(\xi)=(\mathrm{id} \otimes \xi)(r)$.

Let $f, g \in S\left(\mathfrak{g}^{*}\right)^{\mathfrak{g}}$ have degrees $k$ and $\ell$. Write

$$
f=\sum_{\alpha} a_{1}^{\alpha} \cdots a_{k}^{\alpha}, \quad g=\sum_{\beta} b_{1}^{\beta} \cdots b_{\ell}^{\beta} .
$$


Then

$$
\begin{aligned}
\{f, g\}=\sum_{\beta} \sum_{j=1}^{\ell} b_{1}^{\beta} \cdots \check{b}_{j}^{\beta} \cdots b_{\ell}^{\beta} \operatorname{ad}^{*}\left(R\left(b_{j}^{\beta}\right)\right)(f) & \\
& \quad-\sum_{\alpha} \sum_{i=1}^{k} a_{1}^{\alpha} \cdots \check{a}_{i}^{\alpha} \cdots a_{k}^{\alpha} \operatorname{ad}^{*}\left(R\left(a_{i}^{\alpha}\right)\right)(g) .
\end{aligned}
$$

When $f$ and $g$ are both invariant, this bracket vanishes.

We now prove that $S\left(\mathfrak{g}^{*}\right)^{\mathfrak{g}} \subset S\left(\mathfrak{g}^{*}\right)$ is also the associated graded of an inclusion of noncommutative algebras with an increasing filtration:

Theorem 4.3. There exists a morphism of filtered algebras:

$$
\theta: S\left(\mathfrak{g}^{*}\right)^{\mathfrak{g}} \rightarrow U\left(\mathfrak{g}^{*}\right)
$$

where the associated graded morphism is the canonical inclusion $S\left(\mathfrak{g}^{*}\right)^{\mathfrak{g}} \subset S\left(\mathfrak{g}^{*}\right)$. Proof. We denote by FSHA the category of formal series Hopf (FSH) algebras and by FilAlg the category of filtered algebras. There is a contravariant functor (restricted duality) FSHA $\rightarrow$ FilAlg, defined by $0 \mapsto 0^{\circ}$, where

$$
0^{\circ}=\left\{\ell \in 0^{*} \mid \ell\left(\mathfrak{m}^{n}\right)=0 \text { for some } n \geq 0\right\} \subset 0^{*} ;
$$

here $\mathfrak{m} \subset \mathbb{O}$ is the maximal ideal of 0 . The algebra structure of $0^{\circ}$ is defined by

$$
\left(\ell_{1} \cdot \ell_{2}\right)(f)=\left(\ell_{1} \otimes \ell_{2}\right)(\Delta(f))
$$

its filtration is defined by $\left(\mathcal{O}^{\circ}\right)_{\leq n}=\left\{\ell \in \mathcal{O}^{*} \mid \ell\left(\mathfrak{m}^{n+1}\right)=0\right\}$.

We have a category equivalence FSHA $\rightarrow$ LCA, where LCA is the category of Lie coalgebras, taking $O$ to $\mathfrak{m} / \mathrm{m}^{2}$, equipped with the cobracket induced by $\Delta-\Delta^{2,1}$. Then the composed functor LCA $\rightarrow$ FSHA $\rightarrow$ FilAlg is $\mathfrak{c} \mapsto U\left(\mathfrak{c}^{*}\right)$ (recall that $\mathrm{c}^{*}$ is a Lie algebra).

$\left(\mathcal{O}_{\mathfrak{g}^{*}}, \Delta_{0}\right)=\left(\widehat{S}(\mathfrak{g}), \Delta_{0}\right)$ is a graded FSH algebra. Its restricted dual is the graded algebra $S\left(\mathfrak{g}^{*}\right)$. Recall that $\mathrm{O}_{\mathfrak{g}^{*}}$ is also a Poisson algebra. We define the set of Poisson traces on $O_{\mathfrak{g}^{*}}$ as the subspace of all $\ell \in \mathcal{O}_{\mathfrak{g}^{*}}^{\circ}$, such that $\ell(\{u, v\})=0$ for any $u, v \in \widehat{O}_{\mathfrak{g}^{*}}$. Then $\left\{\right.$ Poisson traces on $\left.\mathfrak{O}_{\mathfrak{g}^{*}}\right\} \subset \mathrm{O}_{\mathfrak{g}^{*}}^{\circ}$ identifies with $S\left(\mathfrak{g}^{*}\right)^{\mathfrak{g}} \subset S\left(\mathfrak{g}^{*}\right)$; this is a graded subalgebra of $\mathscr{O}_{\mathfrak{g}^{*}}^{\circ}$. This defines a graded algebra structure on \{Poisson traces on $\mathbb{O}_{\mathfrak{g}^{*}}$.

Consider the FSH algebra $\left(0_{\mathfrak{g}^{*}},{ }^{\rho} \Delta_{0}\right)$. It is isomorphic (as a filtered vector space) to $\left(\mathcal{O}_{\mathfrak{g}^{*}}, \Delta_{0}\right)$, and this isomorphism induces an algebra isomorphism between their associated graded FSH algebras. It follows that we have an isomorphism of filtered vector spaces between the filtered algebra $\left(0_{\mathfrak{g}^{*}},{ }^{\rho} \Delta_{0}\right)^{\circ}$ and $S\left(\mathfrak{g}^{*}\right)$, and the associated graded of this morphism is an algebra isomorphism $\operatorname{gr}\left(\left(\mathcal{O}_{\mathfrak{g}^{*}},{ }^{\rho} \Delta_{0}\right)^{\circ}\right) \rightarrow S\left(\mathfrak{g}^{*}\right)$.

Recall that the vector spaces underlying $\left(0_{\mathfrak{g}^{*}}, \Delta_{0}\right)^{\circ}$ and $\left(\mathcal{O}_{\mathfrak{g}^{*}},{ }^{\rho} \Delta_{0}\right)^{\circ}$ are the same, namely, $\mathscr{O}_{\mathfrak{g}^{*}}^{\circ}$. We claim that the canonical inclusion 


\section{$\left\{\right.$ Poisson traces on $\left.\mathbb{O}_{\mathfrak{g}^{*}}\right\} \subset\left(\mathbb{O}_{\mathfrak{g}^{*}},{ }^{\rho} \Delta_{0}\right)^{\circ}$}

is a morphism of filtered algebras. Indeed, denote by $\cdot_{\rho}$ the product in $\left(0_{\mathfrak{g}^{*}},{ }^{\rho} \Delta_{0}\right)^{\circ}$ and by $\cdot$ the product in $\left(0_{\mathfrak{g}^{*}}, \Delta_{0}\right)^{\circ}$. Let $\ell_{1}, \ell_{2}$ be Poisson traces on $O_{\mathfrak{g}^{*}}$. Then for any $x \in \mathcal{O}_{\mathfrak{g}^{*}}$, we have $\left(\ell_{1} \cdot \ell_{2}\right)(x)=\left(\ell_{1} \otimes \ell_{2}\right)\left(\rho \star \Delta_{0}(f) \star(-\rho)\right)$. Now Leibniz's rule implies that $\left(\ell_{1} \otimes \ell_{2}\right)(\{u, v\})=0$ for any $u, v \in \mathcal{O}_{\mathfrak{g}^{*}}^{\widehat{\otimes} 2}$, therefore $\left(\ell_{1} \cdot \rho \ell_{2}\right)(x)=$ $\left(\ell_{1} \otimes \ell_{2}\right)\left(\Delta_{0}(x)\right)=\left(\ell_{1} \cdot \ell_{2}\right)(x)$. So \{Poisson traces on $\left.O_{\mathfrak{g}^{*}}\right\} \subset\left(O_{\mathfrak{g}^{*}},{ }^{\rho} \Delta_{0}\right)^{\circ}$ is an algebra morphism. Since the filtrations on the vector spaces underlying $\left(0_{\mathfrak{g}^{*}}, \Delta_{0}\right)^{\circ}$ and $\left(\mathcal{O}_{\mathfrak{g}^{*}},{ }^{\rho} \Delta_{0}\right)^{\circ}$ are the same, and since the filtration on \{Poisson traces on $\left.\mathscr{O}_{\mathfrak{g}^{*}}\right\}$ is induced by that of $\left(\mathrm{O}_{\mathfrak{g}^{*}}, \Delta_{0}\right)^{\circ}$, this morphism is filtered, and its associated graded is the canonical inclusion $S\left(\mathfrak{g}^{*}\right)^{\mathfrak{g}} \subset S\left(\mathfrak{g}^{*}\right)$.

Now the FSH algebra isomorphism $\mathscr{O}_{G^{*}} \simeq\left(\mathcal{O}_{\mathfrak{g}^{*}},{ }^{\rho} \Delta_{0}\right)$ (Corollary 3.1) induces a filtered algebra isomorphism $\left(\mathcal{O}_{\mathfrak{g}^{*}},{ }^{\rho} \Delta_{0}\right)^{\circ} \rightarrow \mathcal{O}_{G^{*}}^{\circ}=U\left(\mathfrak{g}^{*}\right)$. The fact that the associated graded of this morphism is the canonical isomorphism $S\left(\mathfrak{g}^{*}\right) \rightarrow \operatorname{gr}\left(U\left(\mathfrak{g}^{*}\right)\right)$ follows from the fact that the completed graded of the FSH algebras $\mathrm{O}_{G^{*}}$ and $\left(\mathbb{O}_{\mathfrak{g}^{*}},{ }^{\rho} \Delta_{0}\right)$ are both $\left(\mathcal{O}_{\mathfrak{g}^{*}}, \Delta_{0}\right)$.

We now compose the filtered algebra morphism \{Poisson traces on $\mathcal{O}_{\mathfrak{g}^{*}}$ \} $\left(0_{\mathfrak{g}^{*}},{ }^{\rho} \Delta_{0}\right)^{\circ}$ with the filtered algebra isomorphism $\left(\mathcal{O}_{\mathfrak{g}^{*}},{ }^{\rho} \Delta_{0}\right)^{\circ} \rightarrow \mathcal{O}_{G^{*}}^{\circ}$, and obtain a filtered algebra morphism $S\left(\mathfrak{g}^{*}\right)^{\mathfrak{g}} \rightarrow U\left(\mathfrak{g}^{*}\right)$, whose associated graded is the canonical inclusion $S\left(\mathfrak{g}^{*}\right)^{\mathfrak{g}} \subset S\left(\mathfrak{g}^{*}\right)$.

The situation may be summarized thus:

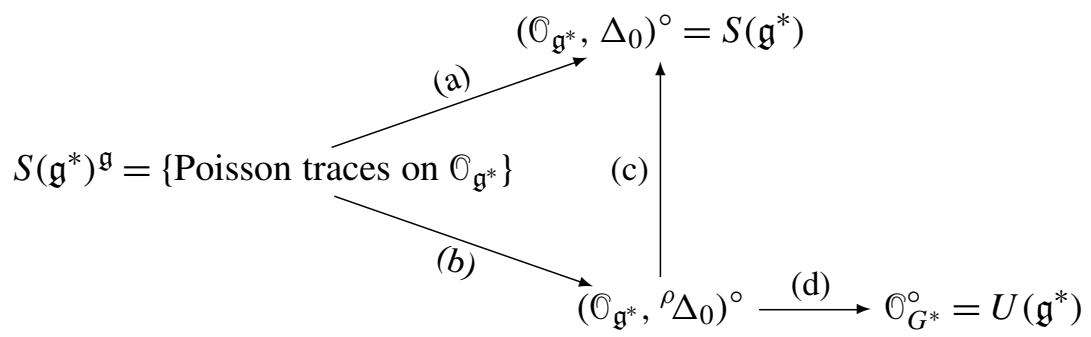

Here $S\left(\mathfrak{g}^{*}\right)^{\mathfrak{g}}$ and $S\left(\mathfrak{g}^{*}\right)$ are graded algebras, $\left(\mathcal{O}_{\mathfrak{g}^{*}},{ }^{\rho} \Delta_{0}\right)^{\circ}$ and $0_{G^{*}}^{\circ}$ are filtered algebras; (a) is a morphism of graded algebras, (c) is an isomorphism of filtered vector spaces, and (b) and (d) are morphisms of filtered algebras, (d) being an isomorphism. The associated graded of (c) is an isomorphism of graded algebras.

Remark 4.4. The restricted dual of the isomorphism $O_{\mathfrak{g}^{*}} \rightarrow \mathcal{O}_{G^{*}}$ appearing in the proof above is an isomorphism of filtered vector spaces $\sigma: S\left(\mathfrak{g}^{*}\right) \rightarrow U\left(\mathfrak{g}^{*}\right)$, whose associated graded is the canonical isomorphism $S\left(\mathfrak{g}^{*}\right) \rightarrow \operatorname{gr}\left(U\left(\mathfrak{g}^{*}\right)\right)$. These properties are also satisfied by the symmetrization map Sym, however $\sigma$ depends on $\rho$, so in general Sym and $\sigma$ are different. 
Remark 4.5. One can check that the morphism $\theta$ is independent of the choice of $(\rho, \varphi)$ (these choices are described in Remark 2.4 and Theorem 2.3).

\section{Duality of QUE and QFSH algebras}

We now recall some facts from [Drinfeld 1987]; proofs can be found in [Gavarini 2002]. We denote by QUE the category of quantized universal enveloping algebras and by QFSH the category of quantized formal series Hopf algebras. We denote by $\mathrm{QUE}_{\mathrm{fd}}$ and $\mathrm{QFSH}_{\mathrm{fd}}$ the subcategories corresponding to finite dimensional Lie bialgebras.

We have the contravariant functors $\mathrm{QUE}_{\mathrm{fd}} \rightarrow \mathrm{QFSH}_{\mathrm{fd}}$ sending $U$ to $U^{*}$ and $\mathrm{QFSH}_{\mathrm{fd}} \rightarrow \mathrm{QUE}_{\mathrm{fd}}$ sending 0 to $0^{\circ}$. These functors are inverse to each other. $U^{*}$ is the full topological dual of $U$ (the space of all ( $\hbar$-adically) continuous $\mathbb{K} \llbracket \hbar \rrbracket-$ linear maps $U \rightarrow \mathbb{K} \llbracket \hbar \rrbracket)$, and $0^{\circ}$ is the space of continuous $\mathbb{K} \llbracket \hbar \rrbracket$-linear forms $\mathbb{O} \rightarrow \mathbb{K} \llbracket \hbar \rrbracket$, where $\mathbb{O}$ is equipped with the $\mathfrak{m}$-adic topology (here $\mathfrak{m} \subset \mathbb{O}$ is the the kernel of $O \rightarrow \mathbb{K}$, that is, the maximal ideal of $O$ ).

We also have covariant functors QUE $\rightarrow$ QFSH, $U \mapsto U^{\prime}$ and QFSH $\rightarrow$ QUE, $\mathrm{O} \mapsto \mathrm{O}^{\vee}$. These functors are also inverse to each other. $U^{\prime}$ is the subalgebra of $U$ defined by

$$
U^{\prime}=\left\{x \in U \mid(\mathrm{id}-\eta \circ \varepsilon)^{\otimes k} \circ \Delta^{(k)}(x) \in \hbar^{k} U^{\widehat{\otimes} k} \text { for all } k \geq 0\right\},
$$

where $\varepsilon$ and $\eta$ are the counit and unit of $U, \Delta^{(1)}=\mathrm{id}, \Delta^{(2)}=\Delta$ is its coproduct, and

$$
\Delta^{(k)}=\left(\Delta \otimes \mathrm{id}^{\otimes k-2}\right) \circ \cdots \circ \Delta .
$$

On the other hand, $\mathcal{O}^{\vee}$ is the $\hbar$-adic completion of

$$
\sum_{k \geq 0} \hbar^{-k} \mathfrak{m}^{k} \subset \mathcal{O}[1 / \hbar]
$$

We also have canonical isomorphisms $\left(U^{\prime}\right)^{\circ} \simeq\left(U^{*}\right)^{\vee}$ and $\left(\mathcal{O}^{\vee}\right)^{*} \simeq\left(\mathcal{O}^{\circ}\right)^{\prime}$.

If $\mathfrak{a}$ is a finite dimensional Lie bialgebra and $U=U_{\hbar}(\mathfrak{a})$ is a QUE algebra quantizing $\mathfrak{a}$, then $U^{*}=\mathrm{O}_{A, \hbar}$ is a QFSH algebra quantizing the Poisson-Lie group $A$ (with Lie bialgebra $\mathfrak{a}$ ), and $U^{\prime}=0_{A^{*}, \hbar}$ is a QFSH algebra quantizing the PoissonLie group $A^{*}$ (with Lie bialgebra $\mathfrak{a}^{*}$ ). If now $\mathrm{O}=\mathrm{O}_{A, \hbar}$ is a QFSH algebra quantizing $A$, then $0^{\circ}=U_{\hbar}(\mathfrak{a})$ is a QUE algebra quantizing $\mathfrak{a}$, and $O^{\vee}=U_{\hbar}\left(\mathfrak{a}^{*}\right)$ is a QFSH algebra quantizing $\mathfrak{a}^{*}$.

We now compute these functors explicitly in the case of cocommutative QUE and commutative QFSH algebras. If $U=U(\mathfrak{a}) \llbracket \hbar \rrbracket$ has a cocommutative coproduct (where $\mathfrak{a}$ is a Lie algebra), then $U^{\prime}$ is a completion of $U(\hbar \mathfrak{a} \llbracket \hbar \rrbracket)$; this is a flat deformation of $\widehat{S}(\mathfrak{a})$ equipped with its linear Lie-Poisson structure. If $G$ is a formal group with function ring $O_{G}$, then $O:=\mathscr{O}_{G} \llbracket \hbar \rrbracket$ is a QFSH algebra, and $\mathcal{O}^{\vee}$ is a 
commutative QUE algebra; it is a quantization of $\left(S\left(\mathfrak{g}^{*}\right)\right.$, commutative product, cocommutative coproduct, co-Poisson structure induced by the Lie bracket of $\mathfrak{g}$ ).

\section{Relation between twist quantization and its functional version}

We define a twist quantization of the coboundary Lie bialgebra $(\mathfrak{g}, r, Z)$ as a pair $(J, \Phi)$, where $J \in U(\mathfrak{g})^{\otimes 2} \llbracket \hbar \rrbracket, \Phi \in U(\mathfrak{g})^{\otimes 3} \llbracket \hbar \rrbracket, \Phi$ is invariant, $(J, \Phi)$ satisfies the twisted cocycle relation

$$
J^{1,2} J^{12,3}=J^{2,3} J^{1,23} \Phi,
$$

and moreover $(\varepsilon \otimes \mathrm{id})(J)=(\mathrm{id} \otimes \varepsilon)(J)=1, J=1^{\otimes 2}+O(\hbar), \Phi=1^{\otimes 3}+O(\hbar)$, $\operatorname{Alt}\left(\left(J-1^{\otimes 2}\right) / \hbar\right)=r+O(\hbar)$, and $\operatorname{Alt}\left(\left(\Phi-1^{\otimes 3}\right) / \hbar^{2}\right)=Z+O(\hbar)$. These conditions imply that $\Phi$ satisfies the pentagon relation, as well as $\varepsilon^{(i)}(\Phi)=1^{\otimes 2}$ for $i=1,2,3$. (We know that such a twist quantization always exists when $\mathfrak{g}$ is triangular or quasitriangular.) Our purpose is to relate twist quantization with its functional version.

The first step is to show that $(J, \Phi)$ can be transformed into an admissible pair, in a sense which we now make precise.

Definition 6.1. An element $x$ in a $Q U E$ algebra $U$ is admissible if $x \in 1+\hbar U$ and if $\hbar \log x$ is in $U^{\prime} \subset U$.

We will use the isomorphism $U(\mathfrak{g})^{\otimes k} \llbracket \hbar \rrbracket \simeq U\left(\mathfrak{g}^{\oplus k}\right) \llbracket \hbar \rrbracket$ to view $U(\mathfrak{g})^{\otimes k} \llbracket \hbar \rrbracket$ as a QUE algebra.

Proposition 6.2. Any twist quantization $(J, \Phi)$ of a coboundary Lie bialgebra $(\mathfrak{g}, r, Z)$ is gauge equivalent to an admissible twist quantization $\left(J^{\prime}, \Phi^{\prime}\right)$ (i.e., such that $J^{\prime}$ and $\Phi^{\prime}$ are admissible).

Proof. We set $U=U(\mathfrak{g}) \llbracket \hbar \rrbracket$. According to Proposition A.1, one can find an invariant $F \in U^{\widehat{\otimes} 2}$, such that $F \in 1^{\otimes 2}+\hbar U_{0}^{\widehat{\otimes} 2}$ and $\Phi^{\prime}:={ }^{F} \Phi=F^{2,3} F^{1,23} \Phi\left(F^{1,2} F^{12,3}\right)^{-1}$ is admissible. In particular, $\Phi^{\prime} \in 1^{\otimes 3}+\hbar^{2} U_{0}^{\widehat{\otimes} 3}$.

Then if we set $J_{0}:=J F$, we have $J_{0}^{1,2} J_{0}^{12,3}=J_{0}^{2,3} J_{0}^{1,23} \Phi^{\prime}$ and $J_{0} \in 1^{\otimes 2}+\hbar U_{0}^{\widehat{\otimes} 2}$. For any $u \in 1^{\otimes 3}+\hbar U_{0}$, define ${ }^{u} J_{0}:=u^{1} u^{2} J_{0}\left(u^{12}\right)^{-1}$. Then $\left({ }^{u} J_{0}, \Phi^{\prime}\right)$ is a twist quantization of $(\mathfrak{g}, r, Z)$. It remains to find $u$ such that $J^{\prime}:={ }^{u} J_{0}$ is admissible.

We will construct $u$ as a product $\cdots u_{2} u_{1}$, where $u_{n} \in 1+\hbar^{n} U_{0}$, in such a way that if $J_{n}:={ }^{u_{n} \cdots u_{1}} J_{0}$, then $\hbar \log J_{n} \in U_{0}^{\prime \widehat{\otimes} 2}+\hbar^{n+2} U_{0}^{\widehat{\otimes} 2}$.

We have already $\hbar \log J_{0} \in \hbar^{2} U_{0}^{\widehat{\otimes} 2}$.

Expand $J_{0}=1^{\otimes 2}+\hbar j_{1}+\cdots$; then $\operatorname{Alt}\left(j_{1}\right)=r$. Moreover, the coefficient of $\hbar$ in $J_{0}^{1,2} J_{0}^{12,3}=J_{0}^{2,3} J_{0}^{1,23} \Phi$ yields $d\left(j_{1}\right)=0$, where $d: U(\mathfrak{g})_{0}^{\otimes 2} \rightarrow U(\mathfrak{g})_{0}^{\otimes 3}$ is the Cartier differential. It follows that for some $a_{1} \in U(\mathfrak{g})_{0}$, we have $j_{1}=r+d\left(a_{1}\right)$. Then if we set $u_{1}:=\exp \left(\hbar a_{1}\right)$ and $J_{1}={ }^{u_{1}} J_{0}$, we get $J_{1} \in 1^{\otimes 2}+\hbar r+\hbar^{2} U_{0}^{\widehat{\otimes} 2}$. Then $\hbar \log J_{1} \in \hbar^{2} r+\hbar^{3} U_{0}^{\widehat{\otimes} 2} \subset U_{0}^{\prime \widehat{\otimes} 2}+\hbar^{3} U_{0}^{\widehat{\otimes} 3}$. 
Assume that for $n \geq 2$, we have constructed $u_{1}, \ldots, u_{n-1}$ such that $\alpha_{n-1}:=$ $\hbar \log \left(J_{n-1}\right) \in U_{0}^{\prime \widehat{\otimes} 2}+\hbar^{n+1} U_{0}^{\widehat{\otimes} 2}$.

We denote by $\bar{\alpha}$ the image of the class of $\alpha_{n-1}$ in $U(\mathfrak{g})_{0}^{\otimes 2} /\left(U(\mathfrak{g})_{0}^{\otimes 2}\right)_{\leq n+1}$ under the isomorphism of this space with $\left(U_{0}^{\prime \widehat{\otimes} 2}+\hbar^{n+1} U_{0}^{\widehat{\otimes} 2}\right) /\left(U_{0}^{\prime \widehat{\otimes} 2}+\hbar^{n+2} U_{0}^{\widehat{\otimes} 2}\right.$ ) (see Lemma A.2). Let $\alpha \in U(\mathfrak{g})_{0}^{\otimes 2}$ be a representative of $\bar{\alpha}$, then $\alpha_{n-1}=\alpha^{\prime}+\hbar^{n+1} \alpha$, where $\alpha^{\prime} \in U_{0}^{\prime \widehat{\otimes} 2}+\hbar^{n+2} U_{0}^{\widehat{\otimes} 2}$. We set $\varphi^{\prime}:=\hbar \log \Phi^{\prime}$; then the twist equation gives $\left(-\alpha^{\prime}-\hbar^{n+1} \alpha\right)^{1,23} \star_{\hbar}\left(-\alpha^{\prime}-\hbar^{n+1} \alpha\right)^{2,3} \star_{\hbar}\left(\alpha^{\prime}+\hbar^{n+1} \alpha\right)^{1,2} \star_{\hbar}\left(\alpha^{\prime}+\hbar^{n+1} \alpha\right)^{12,3}=\varphi^{\prime}$, where $\star_{\hbar}$ is defined as in the Appendix. According to Lemma A.3, the image of this equality in

$$
\left(U^{\widehat{\otimes} 3}+\hbar^{n+1} U^{\prime \widehat{\otimes} 3}\right) /\left(U^{\widehat{\otimes} 3}+\hbar^{n+2} U^{\prime \widehat{\otimes} 3}\right) \simeq U(\mathfrak{g})^{\otimes 3} /\left(U(\mathfrak{g})^{\otimes 3}\right)_{\leq n+1}
$$

is $d(\bar{\alpha})$, where $d$ is the Cartier differential on $U(\mathfrak{g})_{0}^{\otimes \cdot} /\left(U(\mathfrak{g})_{0}^{\otimes \cdot}\right)_{\leq n+1}$. Since $n \geq 2$, the relevant cohomology group vanishes, so

$$
\bar{\alpha}=d(\bar{\beta}), \quad \text { where } \bar{\beta} \in U(\mathfrak{g})_{0} /\left(U(\mathfrak{g})_{0}\right)_{\leq n+1} .
$$

Let $\beta \in U(\mathfrak{g})_{0}$ be a representative of $\bar{\beta}$, and set $u_{n}:=\exp \left(\hbar^{n} \beta\right), J_{n}:={ }^{u_{n}} J_{n-1}$, and $\alpha_{n}:=\hbar \log J_{n}$. Then

$$
\alpha_{n}=\left(\hbar^{n+1} \beta\right)^{1} \star_{\hbar}\left(\hbar^{n+1} \beta\right)^{2} \star_{\hbar} \alpha_{n-1} \star_{\hbar}\left(-\hbar^{n+1} \beta\right)^{12} .
$$

According to Lemma A.3, the image of $\alpha_{n}$ in

$$
\left(U_{0}^{\widehat{\otimes} 2}+\hbar^{n+1} U_{0}^{\prime \widehat{\otimes} 2}\right) /\left(U_{0}^{\widehat{\otimes} 2}+\hbar^{n+2} U_{0}^{\prime \widehat{\otimes} 2}\right) \simeq U(\mathfrak{g})_{0}^{\otimes 2} /\left(U(\mathfrak{g})_{0}^{\otimes 2}\right)_{\leq n+1}
$$

is $\bar{\alpha}-d(\bar{\beta})=0$. So $\alpha_{n}$ belongs to $U_{0}^{\widehat{\otimes} 2}+\hbar^{n+2} U_{0}^{\prime \widehat{\otimes} 2}$, as required. This proves the induction step.

If now $\left(J^{\prime}, \Phi^{\prime}\right)$ is an admissible twist quantization, then $\rho:=\hbar \log J_{\mid \hbar=0}^{\prime}$ and $\varphi:=\hbar \log \Phi_{\mid \hbar=0}^{\prime}$ are formal functions on $\mathfrak{m}_{\mathfrak{g}^{*}}^{\widehat{\otimes} 2}$ and $\mathfrak{m}_{\mathfrak{g}^{*}} \widehat{\otimes}$, solutions of the functional twist equation.

\section{Quantization of $\mathcal{O}_{G}^{\mathfrak{g}} \subset \mathrm{O}_{G}$}

Using a (not necessarily admissible) twist quantization, we construct a formal noncommutative deformation of the inclusion of algebras of Lemma 4.1:

Proposition 7.1. We have an injective algebra morphism $\mathcal{O}_{G}^{\mathfrak{g}} \llbracket \hbar \rrbracket \hookrightarrow \mathcal{O}_{G, \hbar}$ deforming ${O_{G}}_{G}^{\mathfrak{g}} \subset \mathrm{O}_{G}$, where $\mathrm{O}_{G, \hbar}$ is a quantization of the PFSH algebra $\mathscr{O}_{G}$, and $\mathscr{O}_{G}^{\mathfrak{g}} \llbracket \hbar \rrbracket$ is the trivial deformation of the commutative algebra $0_{G}^{\mathfrak{g}}$ (it is also commutative).

Proof. We first construct the QFSH algebra $\mathscr{O}_{G, \hbar}$. For $x \in U(\mathfrak{g}) \llbracket \hbar \rrbracket$, set ${ }^{J} \Delta_{0}(x)=$ $J \Delta_{0}(x) J^{-1}$, where $\Delta_{0}$ is the usual cocommutative coproduct. Then $U_{\hbar}(\mathfrak{g})=$ 
$\left(U(\mathfrak{g}) \llbracket \hbar \rrbracket, m_{0},{ }^{J} \Delta_{0}\right)$ is a quantization of the Lie bialgebra $\mathfrak{g}$ (here $m_{0}$ is the product on $U(\mathfrak{g}))$. The dual $\mathscr{O}_{G, \hbar}:=U_{\hbar}(\mathfrak{g})^{*}$ of this QUE algebra is a QFSH algebra quantizing the PFSH algebra $\mathrm{O}_{G}$. The product in this QFSH algebra is defined by $(f \star g)(x)=(f \otimes g)\left(J \Delta_{0}(x) J^{-1}\right)$ for $f, g \in U_{\hbar}(\mathfrak{g})^{*}$ and $x \in U_{\hbar}(\mathfrak{g})$.

On the other hand, the FSH algebra $\mathrm{O}_{G}$ is equal to $U(\mathfrak{g})^{*}$, and its product is defined by $(f g)(x)=(f \otimes g)\left(\Delta_{0}(x)\right)$ for $f, g \in U(\mathfrak{g})^{*}$ and $x \in U(\mathfrak{g})$.

We say that $f \in U(\mathfrak{g})^{*}$ is a trace if and only if $f(x y)=f(y x)$ for any $x, y \in U(\mathfrak{g})$. Then the inclusion $\{$ traces on $U(\mathfrak{g})\} \subset U(\mathfrak{g})^{*}$ identifies with $\mathcal{O}_{G}^{\mathfrak{g}} \subset \mathfrak{O}_{G}$. In the same way, we define $\{$ traces on $U(\mathfrak{g}) \llbracket \hbar \rrbracket\}$; this is a subalgebra of $U(\mathfrak{g}) \llbracket \hbar \rrbracket^{*} \simeq \mathfrak{O}_{G} \llbracket \hbar \rrbracket$, which identifies with $\mathfrak{O}_{G}^{\mathfrak{g}} \llbracket \hbar \rrbracket$.

The canonical map $\{$ traces on $U(\mathfrak{g}) \llbracket \hbar \rrbracket\} \rightarrow U_{\hbar}(\mathfrak{g})^{*}$ is an algebra morphism. Indeed, if $f_{1}, f_{2}$ are traces on $U(\mathfrak{g}) \llbracket \hbar \rrbracket$, then $f_{1} \otimes f_{2}$ is a trace on $U(\mathfrak{g})^{\otimes 2} \llbracket \hbar \rrbracket$, so $\left(f_{1} \star f_{2}\right)(x)=\left(f_{1} \otimes f_{2}\right)\left(J \Delta_{0}(x) J^{-1}\right)=\left(f_{1} \otimes f_{2}\right)\left(\Delta_{0}(x)\right)=\left(f_{1} f_{2}\right)(x)$ for any $x \in U(\mathfrak{g}) \llbracket \hbar \rrbracket$, thus $f_{1} \star f_{2}=f_{1} f_{2}$. So we have obtained an algebra morphism $\mathcal{O}_{G}^{\mathfrak{g}} \llbracket \hbar \rrbracket \rightarrow U_{\hbar}(\mathfrak{g})^{*}=\mathscr{O}_{G, \hbar}$. It is clearly a deformation of the canonical inclusion $\mathrm{O}_{G}^{\mathfrak{g}} \subset \mathrm{O}_{G}$.

\section{Quantization of $S\left(\mathfrak{g}^{*}\right)^{\mathfrak{g}} \hookrightarrow U\left(\mathfrak{g}^{*}\right)$}

Assume now that $(J, \Phi)$ is an admissible twist quantization. We construct a formal deformation of the inclusion of algebras of Theorem 4.3.

Theorem 8.1. There is an injective algebra morphism:

$$
\theta_{\hbar}: S\left(\mathfrak{g}^{*}\right)^{\mathfrak{g}} \llbracket \hbar \rrbracket \hookrightarrow U_{\hbar}\left(\mathfrak{g}^{*}\right),
$$

where $U_{\hbar}\left(\mathfrak{g}^{*}\right)$ is a quantization of $\mathfrak{g}^{*}$. Its reduction modulo $\hbar$ coincides with the morphism $S\left(\mathfrak{g}^{*}\right)^{\mathfrak{g}} \hookrightarrow U(\mathfrak{g})$ from Theorem 4.3.

Proof. Recall that $U(\mathfrak{g}) \llbracket \hbar \rrbracket^{\prime}$ is a cocommutative QFSH algebra; we denote by $m_{0}$, $\Delta_{0}$ its product and coproduct.

Since

$$
(\varepsilon \otimes \mathrm{id})(J)=(\mathrm{id} \otimes \varepsilon)(J)=1,
$$

we have $\hbar \log J \in \mathfrak{m}_{0}^{\widehat{\otimes} 2}$, where $\mathfrak{m}_{0} \subset U(\mathfrak{g}) \llbracket \hbar \rrbracket^{\prime}$ is the kernel of the counit. According to [Enriquez and Halbout 2003, Proposition 3.1], this implies that the inner automorphism $z \mapsto J z J^{-1}$ of $U(\mathfrak{g})^{\otimes 2} \llbracket \hbar \rrbracket$ restricts to an automorphism of $U(\mathfrak{g})^{\otimes 2} \llbracket \hbar \rrbracket^{\prime}$.

We then equip $U(\mathfrak{g}) \llbracket \hbar \rrbracket^{\prime}$ with the coproduct ${ }^{J} \Delta: x \mapsto J \Delta_{0}(x) J^{-1}$. Then $\left(U(\mathfrak{g}) \llbracket \hbar \rrbracket^{\prime}, m_{0},{ }^{J} \Delta_{0}\right)$ is a QFSH algebra. Its classical limit is the PFSH algebra $\left(\mathrm{O}_{\mathfrak{g}^{*}}, m_{0}, P,{ }^{\rho} \Delta_{0}\right)$. We have seen that this PSFH algebra is isomorphic to $\mathbb{O}_{G^{*}}$, hence $\left(U(\mathfrak{g}) \llbracket \hbar \rrbracket^{\prime}, m_{0},{ }^{J} \Delta_{0}\right)$ is a quantization of $\mathscr{O}_{G^{*}}$.

It now follows from Section 5 that $\left(U(\mathfrak{g}) \llbracket \hbar \rrbracket^{\prime}, m_{0},{ }^{J} \Delta_{0}\right)^{\circ}$ is a quantization of $U\left(\mathfrak{g}^{*}\right)$, which we denote by $U_{\hbar}\left(\mathfrak{g}^{*}\right)$. 
We say that $\varphi \in\left(U(\mathfrak{g}) \llbracket \hbar \rrbracket^{\prime}\right)^{\circ}$ is a trace if $\varphi(x y)=\varphi(y x)$ for any $x, y \in U(\mathfrak{g}) \llbracket \hbar \rrbracket^{\prime}$. Then $\left\{\right.$ traces on $\left.U(\mathfrak{g}) \llbracket \hbar \rrbracket^{\prime}\right\} \subset\left(U(\mathfrak{g}) \llbracket \hbar \mathbb{1}^{\prime}\right)^{\circ}$ is a subalgebra. Indeed, if $\ell_{1}, \ell_{2}$ are traces, then $\ell_{1} \otimes \ell_{2}$ is also a trace, so for $x, y \in U(\mathfrak{g}) \llbracket \hbar \rrbracket^{\prime}$, we have

$$
\left(\ell_{1} \ell_{2}\right)(x y)=\left(\ell_{1} \otimes \ell_{2}\right)(\Delta(x) \Delta(y))=\left(\ell_{1} \otimes \ell_{2}\right)(\Delta(y) \Delta(x))=\left(\ell_{1} \ell_{2}\right)(y x) .
$$

This inclusion identifies with the inclusion $\left(\mathscr{O}_{G} \llbracket \hbar \rrbracket^{\vee}\right)^{\mathfrak{g}} \subset \mathscr{O}_{G} \llbracket \hbar \rrbracket^{\vee}$. Indeed, the Drinfeld functors have the property that $\left(U^{\prime}\right)^{\circ}=\left(U^{*}\right)^{\vee}$ for any QUE algebra $U$.

Now we show that the map \{traces on $\left.U(\mathfrak{g}) \llbracket \hbar \rrbracket^{\prime}\right\} \subset\left(U(\mathfrak{g}) \llbracket \hbar \rrbracket^{\prime},{ }^{J} \Delta_{0}\right)^{\circ}$ is also an algebra morphism. Indeed, let $\cdot_{J}$ be the product of the latter algebra. If $\ell_{1}, \ell_{2}$ are traces and $x, y \in U(\mathfrak{g}) \llbracket \hbar \rrbracket$, then $\left(\ell_{1} \cdot{ }_{J} \ell_{2}\right)(x)=\left(\ell_{1} \otimes \ell_{2}\right)\left(J \Delta_{0}(x) J^{-1}\right)=$ $\left(\ell_{1} \otimes \ell_{2}\right)\left(\Delta_{0}(x)\right)=\left(\ell_{1} \ell_{2}\right)(x)$, so $\ell_{1} \cdot{ }_{J} \ell_{2}=\ell_{1} \ell_{2}$. So we have constructed an algebra morphism $\left(\mathscr{O}_{G} \llbracket \hbar \rrbracket^{\vee}\right)^{\mathfrak{g}} \rightarrow U_{\hbar}(\mathfrak{g})$. It is clearly a deformation of the morphism constructed in Theorem 4.3.

Recall that $\mathscr{O}_{G} \llbracket \hbar \rrbracket^{\vee}$ is the $\hbar$-adic completion of $\sum_{k \geq 0} \hbar^{-k} \mathfrak{m}_{G}^{k} \subset \mathscr{O}_{G}((\hbar)) .^{3}$ Then $\mathrm{O}_{G} \llbracket \hbar \rrbracket^{\vee}$ is a topologically free $\mathbb{K} \llbracket \hbar \rrbracket$-commutative algebra; its specialization at $\hbar=0$ is $\mathscr{O}_{G} \llbracket \hbar \rrbracket^{\vee} / \hbar \mathscr{O}_{G} \llbracket \hbar \rrbracket^{\vee} \simeq S\left(\mathfrak{g}^{*}\right)$.

The action of $\mathfrak{g}$ on $\mathscr{O}_{G}$ induces an action of $\mathfrak{g}$ on $\mathscr{O}_{G} \llbracket \hbar \rrbracket^{\vee}$. Then $\left(\mathscr{O}_{G} \llbracket \hbar \rrbracket^{\vee}\right)^{\mathfrak{g}}$ is the $\hbar$-adic completion of $\sum_{k>0} \hbar^{-k}\left(\mathfrak{m}_{G}^{k}\right)^{\mathfrak{g}}$. We have an inclusion of topologically free $\mathbb{K} \llbracket \hbar \rrbracket$-algebras $\left(\mathscr{O}_{G} \llbracket \hbar \rrbracket^{\vee}\right)^{\mathfrak{g}} \subset \mathscr{O}_{G} \llbracket \hbar \rrbracket^{\vee}$.

Now the dual of the symmetrization map induces an algebra isomorphism

$$
\widehat{S}\left(\mathfrak{g}^{*}\right)=\mathrm{O}_{\mathfrak{g}} \simeq \mathrm{O}_{G}
$$

(dual to the exponential map $\mathfrak{g} \rightarrow G$ ). This isomorphism induces a $\mathfrak{g}$-equivariant isomorphism of $O_{G} \llbracket \hbar \rrbracket^{\vee}$ with the $\hbar$-adic completion of

$$
\sum_{k \geq 0} \hbar^{-k} \mathfrak{m}_{\mathfrak{g}}^{k} \subset \mathcal{O}_{\mathfrak{g}}((\hbar))
$$

So we have an algebra isomorphism $\mathscr{O}_{G} \llbracket \hbar \rrbracket^{\vee} \simeq S\left(\mathfrak{g}^{*}\right) \llbracket \hbar \rrbracket$. It restricts to an isomorphism $\left.\mathfrak{O}_{G} \llbracket \hbar \rrbracket^{\vee}\right)^{\mathfrak{g}} \simeq S\left(\mathfrak{g}^{*}\right)^{\mathfrak{g}} \llbracket \hbar \rrbracket$.

Composing its inverse with the morphism $\left(\mathscr{O}_{G} \llbracket \hbar \rrbracket^{\vee}\right)^{\mathfrak{g}} \rightarrow U_{\hbar}\left(\mathfrak{g}^{*}\right)$, we get the announced morphism $S\left(\mathfrak{g}^{*}\right)^{\mathfrak{g}} \llbracket \hbar \rrbracket \rightarrow U_{\hbar}\left(\mathfrak{g}^{*}\right)$.

\section{The quasitriangular case}

A quasitriangular Lie bialgebra (QTLBA) is a pair $\left(\mathfrak{g}, r^{\prime}\right)$, where $\mathfrak{g}$ is a Lie algebra and $r^{\prime} \in \mathfrak{g}^{\otimes 2}$ is such that $\mathrm{CYB}\left(r^{\prime}\right)=0$ and $t:=r^{\prime}+r^{\prime 2,1} \in S^{2}(\mathfrak{g})^{\mathfrak{g}}$. Any QTLBA gives rise to a coboundary Lie bialgebra $(\mathfrak{g}, r, Z)$, where $r=\left(r^{\prime}-r^{\prime 2,1}\right) / 2$ and

\footnotetext{
${ }^{3} \mathrm{O}_{G} \llbracket \hbar \rrbracket^{\vee}$ may therefore be viewed as the formal Rees algebra associated to the decreasing filtration $\bigcirc_{G} \supset \mathfrak{m}_{G} \supset \mathfrak{m}_{G}^{2} \cdots$.
} 
$Z=\left[t^{1,2}, t^{2,3}\right] / 4$. We call a QTLBA nondegenerate if $\mathfrak{g}$ is finite dimensional and $t$ is nondegenerate.

Let $D: \mathfrak{g}^{*} \rightarrow \mathfrak{g}^{*}$ be the composition of the Lie cobracket $\delta: \mathfrak{g}^{*} \rightarrow \bigwedge^{2}\left(\mathfrak{g}^{*}\right)$ with the Lie bracket of $\mathfrak{g}^{*}$. It is a derivation and a coderivation, and it induces a derivation of $U\left(\mathfrak{g}^{*}\right)$, which we also denote by $D$ (or sometimes $D_{\mathfrak{g}^{*}}$ ).

Proposition 9.1. For any scalar $s$, the algebra $C_{s}:=\operatorname{Ker}\left(\delta-s(D \otimes \mathrm{id}) \circ \Delta_{0}\right)$ is a commutative subalgebra of $U\left(\mathfrak{g}^{*}\right)$.

Proof. The condition $\ell \in C_{s}$ means that $\ell\left(\{u, v\}-s D^{*}(u) v\right)=0$ for any $u, v \in \mathcal{O}_{G^{*}}$ (here $D^{*}$ is the derivation of $0_{G^{*}}$ dual to the coderivation $D$ ).

Let $\ell_{1}, \ell_{2}$ belong to $C_{s}$. Then for any $u, v \in O_{G^{*}}$,

$$
\begin{aligned}
& \left(\ell_{1} \ell_{2}\right)\left(\{u, v\}-s D^{*}(u) v\right) \\
& =\left(\ell_{1} \otimes \ell_{2}\right)\left(\{\Delta(u), \Delta(v)\}-s \Delta\left(D^{*}(u)\right) \Delta(v)\right) \\
& =\left(\ell_{1} \otimes \ell_{2}\right)\left(\left\{u^{(1)}, v^{(1)}\right\} \otimes u^{(2)} v^{(2)}+u^{(1)} v^{(1)} \otimes\left\{u^{(2)}, v^{(2)}\right\}\right. \\
& \left.\quad-s D^{*}\left(u^{(1)}\right) v^{(1)} \otimes u^{(2)} v^{(2)}-u^{(1)} v^{(1)} \otimes s D^{*}\left(u^{(2)}\right) v^{(2)}\right) \\
& =0
\end{aligned}
$$

hence $\ell_{1} \ell_{2} \in C_{s}$. Here $\Delta$ is the coproduct of $\mathscr{O}_{G^{*}}$.

Moreover, we constructed in [Enriquez et al. 2003] an element $\varrho \in \mathfrak{m}_{G^{*}}^{\widehat{\otimes}^{2}}$, such that $\Delta^{\prime}(u)=\varrho \star \Delta(u) \star(-\varrho)$ for any $u \in \mathscr{O}_{G^{*}}$; if $\left(U_{\hbar}(\mathfrak{g}), \mathscr{R}\right)$ is any quantization of $\left(\mathfrak{g}, r^{\prime}\right)$, then $\hbar \log \mathscr{R} \in \mathfrak{m}_{\hbar}^{\widehat{\otimes} 2}$, where $\mathfrak{m}_{\hbar} \subset U_{\hbar}(\mathfrak{g})^{\prime}$ is the augmentation ideal, and the reduction of $\hbar \log \mathscr{R} \bmod \hbar$ equals $\varrho$. Then it follows from $\left(S^{2} \otimes S^{2}\right)(\mathscr{R})=\mathscr{R}$ that

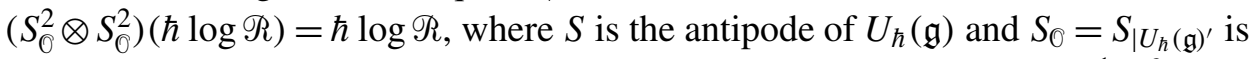
the antipode of $U_{\hbar}(\mathfrak{g})^{\prime} \subset U_{\hbar}(\mathfrak{g})$; since the specialization for $\hbar=0$ of $\hbar^{-1}\left(S_{\overparen{O}}^{2}-\mathrm{id}\right)$ is $D^{*}$, we get $\left(D^{*} \otimes \mathrm{id}+\mathrm{id} \otimes D^{*}\right)(\varrho)=0$.

Then if $\ell_{1}, \ell_{2} \in C_{s}$, then

$$
\begin{aligned}
\left(\ell_{2} \ell_{1}\right)(u) & =\left(\ell_{1} \otimes \ell_{2}\right)\left(\Delta^{\prime}(u)\right)=\left(\ell_{1} \otimes \ell_{2}\right)(\varrho \star \Delta(u) \star(-\varrho)) \\
& =\left(\ell_{1} \otimes \ell_{2}\right)(\Delta(u))+\sum_{n \geq 1}(1 / n !)\left(\ell_{1} \otimes \ell_{2}\right)(\{\varrho,\{\varrho, \ldots,\{\varrho, \Delta(u)\}\}\}) .
\end{aligned}
$$

Now if $f \in O_{G^{*}}^{\widehat{\otimes} 2}$, then $\left(\ell_{1} \otimes \ell_{2}\right)(\{\varrho, f\})=s\left(\ell_{1} \otimes \ell_{2}\right)\left(\left(D^{*} \otimes \mathrm{id}+\mathrm{id} \otimes D^{*}\right)(\varrho) f\right)=0$. It follows that $\ell_{2} \ell_{1}=\ell_{1} \ell_{2}$.

Remark 9.2. If $A$ is a quasitriangular Hopf algebra with antipode $S$, set

$$
C_{s, A}:=\left\{\ell \in A^{*} \mid \ell(a b)=\ell\left(b S^{-2 s}(a)\right) \text { for all } a, b \in A\right\}
$$

for $s \in \mathbb{Z}$. Then it follows from [Drinfeld 1989a] that $C_{s, A}$ is a commutative algebra, and that we have isomorphisms $C_{s} \simeq C_{s+2}$ for any $s \in \mathbb{Z}$. The isomorphism takes 
$\ell \in C_{s}$ to the element $\bar{\ell} \in C_{s+2}$ defined by

$$
\bar{\ell}(x)=\ell\left(x u^{-1} S(u)\right),
$$

where $u=m \circ(\operatorname{id} \otimes S)(R)$; here $m, R$ are the product and $R$-matrix of $A$. The definition of $C_{s, A}$ can be generalized to $s \in \mathbb{K}$ when $A=\left(U_{\hbar}(\mathfrak{g}), \mathscr{R}\right)$ is a quasitriangular QUE Hopf algebra. Define $U_{\hbar}\left(\mathfrak{g}^{*}\right)$ as $\left(U_{\hbar}(\mathfrak{g})^{\prime}\right)^{\circ}=\left(U_{\hbar}(\mathfrak{g})^{*}\right)^{\vee} \supset U_{\hbar}(\mathfrak{g})^{*}$. Then

$$
C_{s, \hbar}:=\left\{\ell \in\left(U_{\hbar}(\mathfrak{g})^{\prime}\right)^{\circ} \mid \ell(a b)=\ell\left(b\left(S^{2}\right)^{-s}(a)\right) \text { for all } a, b \in U_{\hbar}(\mathfrak{g})^{\prime}\right\}
$$

is a commutative subalgebra of $U_{\hbar}\left(\mathfrak{g}^{*}\right)$, and its reduction modulo $\hbar$ is contained in $C_{s}$. In this case, $u^{-1} S(u)$ does not necessarily belong to $U_{\hbar}(\mathfrak{g})^{\prime}$, therefore $C_{s, \hbar}$ and $C_{s+2, \hbar}$ are not necessarily isomorphic.

Remark 9.3. If $(\mathfrak{g}, r, Z)$ is a coboundary Lie bialgebra, then $r$ is $D$-invariant if and only if $(\mu \otimes \mathrm{id})(Z)$ is symmetric (where $\mu$ is the Lie bracket of $\mathfrak{g}$ ). If this is not the case, if we set $\varrho:=\rho^{2,1} \star(-\rho)$, then $\left(D^{*} \otimes \mathrm{id}+\mathrm{id} \otimes D^{*}\right)(\varrho) \neq 0$, so unless $s=0$, one cannot prove that $C_{s}$ is commutative.

For each nondegenerate QTLBA ( $\left.\mathfrak{g}, r^{\prime}\right)$, Semenov-Tian-Shansky [1984] defined an algebra morphism $\Theta: Z(U(\mathfrak{g})) \rightarrow U\left(\mathfrak{g}^{*}\right)$, where $Z(A)$ denotes the center of an algebra $A$. We recall the construction of $\Theta$. There are unique Lie algebra morphisms $L, R: \mathfrak{g}^{*} \rightarrow \mathfrak{g}$, defined by $L(\ell)=(\ell \otimes \mathrm{id})\left(r^{\prime}\right), R(\ell)=-(\mathrm{id} \otimes \ell)\left(r^{\prime}\right)$ for any $\ell \in \mathfrak{g}^{*}$. We denote by $\alpha: U\left(\mathfrak{g}^{*}\right) \rightarrow U(\mathfrak{g})$ the composed map

$$
U\left(\mathfrak{g}^{*}\right) \stackrel{\Delta_{0}}{\rightarrow} U\left(\mathfrak{g}^{*}\right)^{\otimes 2} \stackrel{L \otimes\left(S_{0} \circ R\right)}{\rightarrow} U(\mathfrak{g})^{\otimes 2} \stackrel{m_{0}}{\rightarrow} U(\mathfrak{g}) .
$$

Here $m_{0}, \Delta_{0}$ are the standard product and coproduct maps. We still denote by $L, R$ the algebra morphisms induced by $L, R$, and $S_{0}$ denotes the antipode of $U(\mathfrak{g})$. The associated graded of the map $\alpha$ is the isomorphism $S\left(\mathfrak{g}^{*}\right) \rightarrow S(\mathfrak{g})$ induced by $t$, hence $\alpha$ is an isomorphism. Then $\Theta: Z(U(\mathfrak{g})) \rightarrow U\left(\mathfrak{g}^{*}\right)$ is defined as the restriction of $\alpha^{-1}$ to $Z(U(\mathfrak{g}))$; one can prove that it is an algebra morphism.

We will show, together with Proposition 9.8:

Proposition 9.4. $\operatorname{Im}(\Theta)=C_{1} \subset U\left(\mathfrak{g}^{*}\right)$. The associated graded algebra of $C_{1}$ (for the degree filtration of $\left.U\left(\mathfrak{g}^{*}\right)\right)$ is $S\left(\mathfrak{g}^{*}\right)^{\mathfrak{g}}$.

Remark 9.5. Let $\theta$ be as in Theorem 4.3. The image of $\theta: S\left(\mathfrak{g}^{*}\right)^{\mathfrak{g}} \rightarrow U\left(\mathfrak{g}^{*}\right)$ is \{Poisson traces on $0_{G^{*}}$ \}, that is, $C_{0}=\operatorname{Ker}(\delta)$, where $\delta: U\left(\mathfrak{g}^{*}\right) \rightarrow \bigwedge^{2} U\left(\mathfrak{g}^{*}\right)$ is the co-Poisson map of $U\left(\mathfrak{g}^{*}\right)$. So the images of $\Theta$ and $\theta$ do not necessarily coincide.

Notice that $C_{1}$ is also the image of a morphism $S\left(\mathfrak{g}^{*}\right)^{\mathfrak{g}} \rightarrow U\left(\mathfrak{g}^{*}\right)$, since

$$
Z(U(\mathfrak{g}))=U(\mathfrak{g})^{\mathfrak{g}} \simeq S(\mathfrak{g})^{\mathfrak{g}} \simeq S\left(\mathfrak{g}^{*}\right)^{\mathfrak{g}},
$$


where the second equality is Duflo's isomorphism [1969], and the third equality uses the nondegenerate pairing of $\mathfrak{g}$ dual to $t$. Hence $C_{0}$ and $C_{1}$ are images of morphisms $S\left(\mathfrak{g}^{*}\right)^{\mathfrak{g}} \rightarrow U\left(g^{*}\right)$, whose associated graded is the canonical injection, but which do not necessarily coincide.

We now construct a deformation $\Theta_{\hbar}$ of $\Theta$.

Lemma 9.6 [Drinfeld 1989a]. Let $(A, \Delta, R)$ be a quasitriangular Hopf algebra with antipode $S$. Define a linear map $\alpha_{A}: A^{*} \rightarrow A$ by $\alpha_{A}(\ell)=(\ell \otimes \mathrm{id})\left(R^{21} R\right)$. Then $\alpha_{A}$ induces an algebra morphism $C_{1, A} \rightarrow Z(A)$.

Lemma 9.7. Assume moreover that $A$ is finite dimensional and $R^{2,1} R$ is nondegenerate. Then the map $C_{1} \rightarrow Z(A)$ is a linear isomorphism. Its inverse induces an algebra morphism $\Theta_{A}: Z(A) \rightarrow A^{*}$.

Proof. We have to check that if $\ell \in A^{*}$ is such that $\alpha_{A}(\ell) \in Z(A)$, then $\ell$ is a trace. The condition $\alpha_{A}(\ell) \in Z(A)$ means that for any $a \in A$, we have

$$
(\ell \otimes \mathrm{id})\left(\left[R^{2,1} R, 1 \otimes a\right]\right)=0 .
$$

It follows that

$$
S^{-1}\left(a^{(4)}\right)(\ell \otimes \mathrm{id})\left(\left[R^{2,1} R, a^{(2)} S^{-1}\left(a^{(1)}\right) \otimes a^{(3)}\right]\right)=0
$$

for any $a \in A$. Since $R^{2,1} R$ commutes with the image of $\Delta_{A}$, we have

$$
(\ell \otimes \mathrm{id})\left(\left(a^{(2)} \otimes S^{-1}\left(a^{(4)}\right) a^{(3)}\right)\left[R^{2,1} R, S^{-1}\left(a^{(1)}\right) \otimes 1\right]\right)=0 .
$$

Therefore $(\ell \otimes \mathrm{id})\left(\left(a^{(2)} \otimes 1\right) R^{2,1} R\left(S^{-1}\left(a^{(1)}\right) \otimes \mathrm{id}\right)\right)=\varepsilon(a)(\ell \otimes \mathrm{id})\left(R^{2,1} R\right)$.

Since $R^{2,1} R$ is nondegenerate, this means that $\ell\left(a^{(2)} b S^{-1}\left(a^{(1)}\right)\right)=\varepsilon(a) \ell(b)$ for any $b \in A$. Replacing $a \otimes b$ by $a^{(1)} \otimes S\left(a^{(2)}\right) b$, we get $\ell\left(b S^{-1}(a)\right)=\ell(S(a) b)$, so $\ell \in C_{1}$.

The QUE algebra version of these lemmas is parts (i) and (ii) of the following proposition. Let $\left(\mathfrak{g}, r^{\prime}\right)$ be a QTLBA and let $\left(U_{\hbar}(\mathfrak{g}), \Delta, \mathscr{R}\right)$ be a quantization of $\left(\mathfrak{g}, r^{\prime}\right)$.

Proposition 9.8. (i) The linear map $U_{\hbar}(\mathfrak{g})^{*} \rightarrow U_{\hbar}(\mathfrak{g}), \ell \mapsto(\ell \otimes \mathrm{id})\left(\mathscr{R}^{2,1}\right)$ extends to a map $\alpha_{\hbar}: U_{\hbar}\left(\mathfrak{g}^{*}\right) \rightarrow U_{\hbar}(\mathfrak{g})$.

(ii) If $\left(\mathfrak{g}, r^{\prime}\right)$ is nondegenerate, then $\alpha_{\hbar}$ is a linear isomorphism, and it restricts to an algebra isomorphism $C_{1, \hbar} \rightarrow Z\left(U_{\hbar}(\mathfrak{g})\right)$.

(iii) Proposition 9.4 is true.

Proof. We prove part (i). Define $L_{\hbar}, R_{\hbar}^{\prime}: U_{\hbar}(\mathfrak{g})^{*} \rightarrow U_{\hbar}(\mathfrak{g})$ by

$$
L_{\hbar}(\xi)=(\xi \otimes \mathrm{id})(\mathscr{R}), \quad R_{\hbar}^{\prime}(\xi)=(\mathrm{id} \otimes \xi)(\mathscr{R}) .
$$


According to [Enriquez and Halbout 2003],

$$
\hbar \log \mathscr{R} \subset\left(U_{\hbar}(\mathfrak{g})_{0}^{\prime}\right)^{\widehat{\otimes} 2} \subset U_{\hbar}(\mathfrak{g})_{0}^{\prime} \widehat{\otimes} \hbar U_{\hbar}(\mathfrak{g})_{0},
$$

so that $\log \mathscr{R} \in U_{\hbar}(\mathfrak{g})_{0}^{\prime} \widehat{\otimes} U_{\hbar}(\mathfrak{g})_{0}$. According to [Enriquez et al. 2003, Appendix], the image of $\log \Re$ in $\left(\mathfrak{m}_{G^{*}} / \mathfrak{m}_{G^{*}}^{2}\right) \widehat{\otimes} U(\mathfrak{g})_{0}$ (by reduction mod $\hbar$ followed by projection) is $r^{\prime}$. It follows that $\mathscr{R} \in U_{\hbar}(\mathfrak{g})^{\prime} \widehat{\otimes} U_{\hbar}(\mathfrak{g})$, therefore $L_{\hbar}$ extends to a map $U_{\hbar}\left(\mathfrak{g}^{*}\right) \rightarrow U_{\hbar}(\mathfrak{g})$; this map is necessarily a QUE algebra morphism. The quasitriangularity identities imply that the image of $\mathscr{R}$ in $\widehat{O}_{G^{*}} \widehat{\otimes} U(\mathfrak{g})$ has the form $\exp (\rho)$, where $\rho \in \mathfrak{m}_{G^{*}} \otimes \mathfrak{g}$ is a lift of $r$. It follows that the reduction mod $\hbar$ of $L_{\hbar}$ is the morphism induced by $\mathfrak{g}^{*} \rightarrow \mathfrak{g}, \ell \mapsto(\ell \otimes \mathrm{id})(r)$. In the same way, $R_{\hbar}^{\prime}$ extends to a (anti)morphism $U_{\hbar}\left(\mathfrak{g}^{*}\right) \rightarrow U_{\hbar}(\mathfrak{g})$.

Define $\alpha_{\hbar}: U_{\hbar}\left(\mathfrak{g}^{*}\right) \rightarrow U_{\hbar}(\mathfrak{g})$ by

$$
x \mapsto m \circ\left(L_{\hbar} \otimes R_{\hbar}^{\prime}\right) \circ \Delta .
$$

Then $\alpha_{\hbar}$ extends $\ell \mapsto(\ell \otimes \mathrm{id})\left(\mathscr{R} \mathscr{R}^{2,1}\right)$.

We prove part (ii). The reduction mod $\hbar$ of $\alpha_{\hbar}$ is $\alpha$, which is a linear isomorphism; hence $\alpha_{\hbar}$ is a linear isomorphism. The second part is proved as Lemma 9.6.

We prove Proposition 9.4. Assume that $U_{\hbar}(\mathfrak{g})$ is as in [Etingof and Kazhdan 1996], hence $U_{\hbar}(\mathfrak{g}) \simeq U(\mathfrak{g}) \llbracket \hbar \rrbracket$ as algebras. Then $Z\left(U_{\hbar}(\mathfrak{g})\right) \simeq Z(U(\mathfrak{g})) \llbracket \hbar \rrbracket$. Statement (ii) implies that $\alpha$ induces an isomorphism $(\bmod \hbar)\left(C_{1, \hbar}\right) \rightarrow Z(U(\mathfrak{g}))$; here $(\bmod \hbar)$ is the reduction modulo $\hbar$. On the other hand, $(\bmod \hbar)\left(C_{1, \hbar}\right) \subset C_{1}$, therefore $\Theta(Z(U(\mathfrak{g}))) \subset C_{1}$.

The map

$$
\delta-(D \otimes \mathrm{id}) \circ \Delta_{0}: U\left(\mathfrak{g}^{*}\right) \rightarrow U\left(\mathfrak{g}^{*}\right)^{\otimes 2}
$$

is filtered, and its associated graded is the dual $\delta: S\left(\mathfrak{g}^{*}\right) \rightarrow \bigwedge^{2}\left(S\left(\mathfrak{g}^{*}\right)\right)$ of the Poisson bracket of $S(\mathfrak{g})$. We have a surjective morphism

$$
S(\mathfrak{g})_{\mathfrak{g}}=S(\mathfrak{g}) /\{\mathfrak{g}, S(\mathfrak{g})\} \rightarrow S(\mathfrak{g}) /\{S(\mathfrak{g}), S(\mathfrak{g})\}
$$

to the cokernel of this Poisson bracket, hence $\operatorname{Ker}(\delta) \hookrightarrow\left(S(\mathfrak{g})_{\mathfrak{g}}\right)^{*}=S\left(\mathfrak{g}^{*}\right)^{\mathfrak{g}}$. We have $\operatorname{gr}\left(C_{1}\right) \subset \operatorname{Ker}(\delta)$, hence $\operatorname{gr}\left(C_{1}\right) \subset S\left(\mathfrak{g}^{*}\right)^{\mathfrak{g}}$. Now since $\Theta$ is filtered and its associated graded takes $\operatorname{gr}(Z(U(\mathfrak{g}))) \simeq S(\mathfrak{g})^{\mathfrak{g}}$ to $S\left(\mathfrak{g}^{*}\right)^{\mathfrak{g}}$, we get $\operatorname{gr}\left(C_{1}\right)=S\left(\mathfrak{g}^{*}\right)^{\mathfrak{g}}$ and $\Theta(Z(U(\mathfrak{g})))=C_{1}$.

We denote by $\Theta_{\hbar}: Z\left(U_{\hbar}(\mathfrak{g})\right) \rightarrow U_{\hbar}\left(\mathfrak{g}^{*}\right)$ the algebra morphism inverse to $\alpha_{\hbar}$, which is to say the QUE algebra version of $\Theta_{A}$ defined above.

The image of $\Theta_{\hbar}$ is $C_{1, \hbar}$. When the quantization is as in [Etingof and Kazhdan 1996], $U_{\hbar}(\mathfrak{g}) \simeq U(\mathfrak{g}) \llbracket \hbar \rrbracket$, so this image is not the same as that of $\theta_{\hbar}$, which is \{traces on $\left.U_{\hbar}(\mathfrak{g})^{\prime}\right\}=C_{0, \hbar}$. Therefore in this case, the images of $\theta_{\hbar}$ and $\Theta_{\hbar}$ do not coincide. 


\section{On the canonical derivation of ${ }^{\circ} G^{*}$}

Let $\left(\mathfrak{a}, \mu_{\mathfrak{a}}, \delta_{\mathfrak{a}}\right)$ be a finite dimensional Lie bialgebra. Then $\mathscr{O}_{A}$ is a Poisson-Lie group, dual to $U(\mathfrak{a})$. Set $D_{\mathfrak{a}}:=\mu_{\mathfrak{a}} \circ \delta_{\mathfrak{a}}$, then $D_{\mathfrak{a}}$ is a derivation of $U(\mathfrak{a})$, such that if $U_{\hbar}(\mathfrak{a})$ is any quantization of $U(\mathfrak{a})$ with antipode $S$, then $D_{\mathfrak{a}}=\hbar^{-1}\left(S^{2}-\mathrm{id}\right)_{\mid \hbar=0}$; see [Drinfeld 1989a]. It follows that the dual derivation $D_{\mathfrak{a}}^{*}$ of $\mathscr{O}_{A}$ has the same property.

When $\mathfrak{a}=\left(\mathfrak{g}, r^{\prime}\right)$ is a quasitriangular Lie bialgebra, $D_{\mathfrak{a}}$ is inner, given by $D_{\mathfrak{a}}(x)=$ $-\left[\mu\left(r^{\prime}\right), x\right]$ for any $x \in U(\mathfrak{g})$; here $\mu$ is the Lie bracket of $\mathfrak{g}$ [Drinfeld 1989a].

Proposition 10.1. If $\mathfrak{g}$ is a nondegenerate quasitriangular Lie bialgebra, then the derivation $D_{\mathfrak{g}^{*}}^{*}$ of $\mathrm{O}_{G^{*}}$ is inner, that is, there exists a function $h \in \mathbb{O}_{G^{*}}$ such that $D_{\mathfrak{g}^{*}}^{*}(f)=\{h, f\}$ for any $f \in \mathbb{O}_{G^{*}}$.

Proof. We assume that $\mathfrak{g}$ is the double $\mathfrak{a}_{+} \oplus \mathfrak{a}_{-}$of a Lie bialgebra $\mathfrak{a}_{+}$(here $\mathfrak{a}_{-}=\mathfrak{a}_{+}^{*}$ ); the general case is similar. Then $\mathfrak{g}^{*}$ is (as a Lie algebra) the direct sum $\mathfrak{a}_{+} \oplus \mathfrak{a}_{-}$. Let $A_{ \pm}$be the formal groups corresponding to $\mathfrak{a}_{ \pm}$. The morphism $\alpha: U\left(\mathfrak{g}^{*}\right) \rightarrow$ $U(\mathfrak{g})$ is now $U\left(\mathfrak{a}_{+}\right) \otimes U\left(\mathfrak{a}_{-}\right) \rightarrow U(\mathfrak{g}), x_{+} \otimes x_{-} \mapsto x_{-} S\left(x_{+}\right)$. The dual morphism $\alpha^{*}: \mathbb{O}_{G} \rightarrow \mathbb{O}_{G^{*}}$ takes $F \in \mathscr{O}_{G}$ to $f \in \mathscr{O}_{G^{*}}$ given by $f\left(g_{+}, g_{-}\right):=F\left(g_{-} g_{+}^{-1}\right)$.

Lemma 10.2. Let $D_{\mathfrak{g}}^{*}, D_{\mathfrak{g}^{*}}^{*}$ be the canonical derivations of $\mathrm{O}_{G}$ and $\mathrm{O}_{G^{*}}$. Then $\alpha^{*} \circ D_{\mathfrak{g}}^{*}=D_{\mathfrak{g}^{*}}^{*} \circ \alpha^{*}$. Moreover, $D_{\mathfrak{g}}^{*}=\mathbf{L}_{\mu\left(r^{\prime}\right)}-\mathbf{R}_{\mu\left(r^{\prime}\right)}$, where $\mu$ is the Lie bracket of $\mathfrak{g}$ and $\mathbf{L}_{a} f(g)=(d / d \varepsilon)_{\mid \varepsilon=0} F\left(e^{\varepsilon a} g\right), \mathbf{R}_{a} f(g)=(d / d \varepsilon)_{\mid \varepsilon=0} F\left(g e^{\varepsilon a}\right)$.

Proof. $D_{\mathfrak{g}^{*}}$ is a coderivation, so $\Delta_{0}: U\left(\mathfrak{g}^{*}\right) \rightarrow U\left(\mathfrak{g}^{*}\right)^{\otimes 2}$ intertwines $D_{\mathfrak{g}^{*}}$ and $D_{\mathfrak{g}^{*}} \otimes \mathrm{id}+\mathrm{id} \otimes D_{\mathfrak{g}^{*}} ; L$ and $R$ are Lie bialgebra morphisms, so they intertwine $D_{\mathfrak{g}^{*}}$ and $D_{\mathfrak{g}} ; S$ commutes with $D_{\mathfrak{g}}$; and $D_{\mathfrak{g}}$ is a derivation, so $m_{0}$ intertwines $D_{\mathfrak{g}} \otimes \mathrm{id}+\mathrm{id} \otimes D_{\mathfrak{g}}$ with $D_{\mathfrak{g}}$. Hence $\alpha \circ D_{\mathfrak{g}^{*}}=D_{\mathfrak{g}} \circ \alpha$. The first part follows.

According to [Drinfeld 1989a], $D_{\mathfrak{g}}(x)=-\left[\mu\left(r^{\prime}\right), x\right]$, which implies the second part.

In [Semenov-Tian-Shansky 1985], the image of the Poisson bracket on $G^{*}$ under the formal isomorphism $\alpha: G^{*} \rightarrow G$ dual to $\alpha^{*}$ was computed. Let $f, h \in \mathbb{O}_{G^{*}}$, and $F=\left(\alpha^{*}\right)^{-1}(f), H=\left(\alpha^{*}\right)^{-1}(h)$; then

$$
\begin{aligned}
\left(\alpha^{*}\right)^{-1}(\{f, h\})(g) & \\
= & \left\langle\left(d_{\mathbf{R}}-d_{\mathbf{L}}\right) F(g) \otimes d_{\mathbf{L}} H(g), r^{\prime}\right\rangle+\left\langle\left(d_{\mathbf{R}}-d_{\mathbf{L}}\right) F(g) \otimes d_{\mathbf{R}} H(g),\left(r^{\prime}\right)^{2,1}\right\rangle \\
& =\left\langle\left(d_{\mathbf{L}}-d_{\mathbf{R}}\right) F(g), L\left(d_{\mathbf{R}} H(g)\right)-R\left(d_{\mathbf{L}} H(g)\right)\right\rangle,
\end{aligned}
$$

where $g \in G$ and $d_{\mathbf{L}} F(g), d_{\mathbf{R}} F(g) \in \mathfrak{g}^{*}$ are the left and right differentials defined by $\left\langle d_{\mathbf{L}} F(g), a\right\rangle=\left(\mathbf{L}_{a} F\right)(g),\left\langle d_{\mathbf{R}} F(g), a\right\rangle=\left(\mathbf{R}_{a} F\right)(g)$ for any $a \in \mathfrak{g}$.

Lemma 10.3. There exists a function $H(g) \in \mathscr{O}_{G}$ such that

$$
L\left(d_{\mathbf{R}} H(g)\right)-R\left(d_{\mathbf{L}} H(g)\right)=\mu\left(r^{\prime}\right) .
$$


Proof. We prove this when $\mathfrak{g}$ is the double $\mathfrak{a}_{+} \oplus \mathfrak{a}_{-}$of a Lie bialgebra $\mathfrak{a}_{+}$. Then set $a=\left(a_{+}, a_{-}\right)$, where $a_{ \pm} \in \mathfrak{a}_{ \pm}$. We have $\mathfrak{g}^{*}=\mathfrak{a}_{+} \oplus \mathfrak{a}_{-}$, and we should solve: $d_{\mathbf{R}} H_{a}(g)=\mu\left(r^{\prime}\right)_{-}+u_{+}(g), d_{\mathbf{L}} H_{a}(g)=\mu\left(r^{\prime}\right)_{+}+u_{-}(g)$, where $u_{ \pm}(g)$ are functions $G \rightarrow \mathfrak{a}_{ \pm}$. Now $d_{\mathbf{L}} H(g)=\operatorname{Ad}(g)\left(d_{\mathbf{R}} H(g)\right)$, hence

$$
\mu\left(r^{\prime}\right)_{+}+u_{-}(g)=\operatorname{Ad}(g)\left(\mu\left(r^{\prime}\right)_{-}+u_{+}(g)\right) .
$$

We decompose $g=g_{-} g_{+}^{-1}$, where $g_{ \pm} \in A_{ \pm}=\exp \left(\mathfrak{a}_{ \pm}\right)$; we get

$$
\operatorname{Ad}\left(g_{+}^{-1}\right)\left(u_{+}(g)\right)-\operatorname{Ad}\left(g_{-}^{-1}\right)\left(u_{-}(g)\right)=\operatorname{Ad}\left(g_{-}^{-1}\right)\left(\mu\left(r^{\prime}\right)_{+}\right)-\operatorname{Ad}\left(g_{+}^{-1}\right)\left(\mu\left(r^{\prime}\right)_{-}\right) .
$$

Therefore

$$
u_{+}(g)=\operatorname{Ad}\left(g_{+}\right)\left(\operatorname{Ad}\left(g_{-}^{-1}\right)\left(\mu\left(r^{\prime}\right)_{+}\right)-\operatorname{Ad}\left(g_{+}^{-1}\right)\left(\mu\left(r^{\prime}\right)_{-}\right)\right)_{+},
$$

and the condition is

$$
d_{\mathbf{R}} H(g)=\operatorname{Ad}\left(g_{+}\right)\left(\operatorname{Ad}\left(g_{+}^{-1}\right)\left(\mu\left(r^{\prime}\right)_{-}\right)\right)_{-}+\operatorname{Ad}\left(g_{+}\right)\left(\operatorname{Ad}\left(g_{-}^{-1}\right)\left(\mu\left(r^{\prime}\right)_{+}\right)\right)_{+},
$$

that is,

$$
\begin{aligned}
\mathbf{R}_{\alpha} H_{a}(g) & =\left\langle\mu\left(r^{\prime}\right)_{-}, \operatorname{Ad}\left(g_{+}\right)\left(\left(\operatorname{Ad}\left(g_{+}^{-1}\right)(\alpha)\right)_{+}\right)\right\rangle \\
& +\left\langle\mu\left(r^{\prime}\right)_{+}, \operatorname{Ad}\left(g_{-}\right)\left(\left(\operatorname{Ad}\left(g_{+}^{-1}\right)(\alpha)\right)_{-}\right)\right\rangle
\end{aligned}
$$

for any $\alpha \in \mathfrak{g}$. We denote by $A_{\alpha}(g)$ the right hand side of (10-1).

We compute $\mathbf{R}_{\alpha} A_{\beta}-\mathbf{R}_{\beta} A_{\alpha}$ for $\alpha, \beta \in \mathfrak{g}$. Recall that $g=g_{-} g_{+}^{-1}$; then we have $\mathbf{R}_{\alpha}(g)=g \alpha$, so $\mathbf{R}_{\alpha}\left(g_{ \pm}^{-1}\right)= \pm\left(\operatorname{Ad}\left(g_{ \pm}^{-1}\right)(\alpha)\right)_{ \pm} g_{ \pm}^{-1}$. After computations, we find

$$
\mathbf{R}_{\alpha} A_{\beta}-\mathbf{R}_{\beta} A_{\alpha}=A_{[\beta, \alpha]}+B_{\alpha, \beta},
$$

where

$$
\begin{aligned}
B_{\alpha, \beta}(g)= & -\left\langle\left[\left(\operatorname{Ad}^{*}\left(g_{+}^{-1}\right)(\beta)\right)_{+},\left(\operatorname{Ad}^{*}\left(g_{+}^{-1}\right)(\alpha)\right)_{+}\right],\left(\operatorname{Ad}\left(g_{+}^{-1}\right)\left(\mu\left(r^{\prime}\right)_{-}\right)\right)_{-}\right\rangle \\
& +\left\langle\left[\left(\operatorname{Ad}^{*}\left(g_{+}^{-1}\right)(\beta)\right)_{-},\left(\operatorname{Ad}^{*}\left(g_{+}^{-1}\right)(\alpha)\right)_{-}\right],\left(\operatorname{Ad}\left(g_{-}^{-1}\right)\left(\mu\left(r^{\prime}\right)_{+}\right)\right)_{+}\right\rangle .
\end{aligned}
$$

Now for $u, v \in \mathfrak{a}_{+}$, we have

$$
\begin{aligned}
\left\langle[u, v],\left(\operatorname{Ad}\left(g_{+}^{-1}\right)\left(\mu\left(r^{\prime}\right)_{-}\right)\right)_{-}\right\rangle & =\left\langle[u, v], \operatorname{Ad}\left(g_{+}^{-1}\right)\left(\mu\left(r^{\prime}\right)_{-}\right)\right\rangle \\
& =\left\langle\left[\operatorname{Ad}^{*}\left(g_{+}\right)(u), \operatorname{Ad}^{*}\left(g_{+}\right)(v)\right], \mu\left(r^{\prime}\right)_{-}\right\rangle \\
& =\left\langle\left[\operatorname{Ad}^{*}\left(g_{+}\right)(u), \operatorname{Ad}^{*}\left(g_{+}\right)(v)\right], \mu\left(r^{\prime}\right)\right\rangle \\
& =\left\langle\operatorname{Ad}^{*}\left(g_{+}\right)(u) \otimes \operatorname{Ad}^{*}\left(g_{+}\right)(v), \delta\left(\mu\left(r^{\prime}\right)\right)\right\rangle=0,
\end{aligned}
$$

since $\delta\left(\mu\left(r^{\prime}\right)\right)=0$ [Drinfeld 1989a]. In the same way, the second term of $B_{\alpha, \beta}(g)$ vanishes. Hence the system (10-1) has a solution (it is unique if we impose that $H$ vanishes at the origin). 
We continue the proof of the proposition. If $h=-\alpha^{*}(H)$ with $H$ as in Lemma 10.3 , and for any $f \in \mathbb{O}_{G^{*}}$, we have

$$
\begin{aligned}
D_{\mathfrak{g}^{*}}(f) & =\alpha^{*}\left(D_{\mathfrak{g}}^{*}(F)\right)=\alpha^{*}\left(\left(\mathbf{L}_{\mu\left(r^{\prime}\right)}-\mathbf{R}_{\mu\left(r^{\prime}\right)}\right)(F)\right) \\
& =\alpha^{*}\left(\left\langle\left(d_{\mathbf{L}}-d_{\mathbf{R}}\right)(F)(g), R\left(d_{\mathbf{L}} H(g)\right)-L\left(d_{\mathbf{R}} H(g)\right)\right\rangle\right)=\{h, f\} .
\end{aligned}
$$

\section{Appendix. Proof that associators can be made admissible}

In [Enriquez and Halbout 2004], Proposition 3.2, statement (2) should read "Assume that $x \in U^{\prime}$ and for any trees $R$...". This affects Proposition 4.5 of the same paper, because the proof implicitly relies on the statement of Proposition 3.2 without the assumption $x \in U^{\prime}$. Below we prove a particular case of Proposition 4.5 (the general case is similar).

Proposition A.1. Let $\mathfrak{g}$ be a Lie algebra and let $\Phi \in U(\mathfrak{g})^{\otimes 3} \llbracket \hbar \rrbracket$ be an invariant solution of the pentagon equation (2-1), such that $\varepsilon^{(i)}(\Phi)=1^{\otimes 2}$ for $i=1,2,3$, $\Phi=1^{\otimes 3}+O(\hbar)$ and $\operatorname{Alt}(\Phi)=O\left(\hbar^{2}\right)$. Then there exists an invariant twist $F$ in $U(\mathfrak{g})^{\otimes 2} \llbracket \hbar \rrbracket$ such that $\varepsilon^{(i)}(F)=1$ for $i=1,2, F=1^{\otimes 2}+O(\hbar)$, and

$$
{ }^{F} \Phi=F^{2,3} F^{1,23} \Phi\left(F^{1,2} F^{12,3}\right)^{-1}
$$

is admissible, that is, $\hbar \log F \Phi \in\left(U(\mathfrak{g}) \llbracket \hbar \rrbracket^{\prime}\right)^{\widehat{\otimes} 3}$.

Proof. We will construct $F$ as a product $\cdots F_{2} F_{1}$, where $F_{n}$ belongs to $1^{\otimes 2}+\hbar^{i} U_{0}^{\widehat{\otimes} 2}$ and is such that if $\Phi_{n}:={ }^{F_{n} \cdots F_{1}} \Phi$, then $\hbar \log \Phi_{n} \in\left(U_{0}^{\prime}\right)^{\widehat{\otimes} 2}+\hbar^{n+2} U_{0}^{\widehat{\otimes} 3}$. Here $U=$ $U(\mathfrak{g}) \llbracket \hbar \rrbracket$ and the index 0 denotes the augmentation ideals.

We first construct $F_{1}$. Expand $\Phi=1^{\otimes 3}+\hbar \phi_{1}+\cdots$, then $d\left(\phi_{1}\right)$ and $\operatorname{Alt}\left(\phi_{1}\right)$ vanish, hence $\phi_{1}=d\left(\psi_{1}\right)$, where $\psi_{1} \in\left(U_{0}^{\widehat{\otimes} 2}\right)^{\mathfrak{g}}$ (here $d$ is the Cartier differential of $\left.\left(U_{0}^{\otimes \cdot}\right)^{\mathfrak{g}}\right)$. We then set $F_{1}=1^{\otimes 2}+\hbar \psi_{1}$; we get $\Phi_{1}=1^{\otimes 3}+\hbar^{2} \phi_{2}^{\prime}+\cdots$. Then $\hbar \log \Phi_{1} \in \hbar^{3} U_{0}^{\widehat{\otimes} 3}$.

Now $d\left(\phi_{2}^{\prime}\right)=0$, so there exists $\psi_{2} \in\left(U_{0}^{\widehat{\otimes} 2}\right)^{\mathfrak{g}}$ such that $\phi_{2}^{\prime}=Z+d\left(\psi_{2}\right)$, where $Z \in \bigwedge^{3}(\mathfrak{g}) \llbracket \hbar \rrbracket$. Set $F_{2}:=1^{\otimes 2}+\hbar^{2} \psi_{2}$; we get

$$
\hbar \log \Phi_{2} \in \hbar^{3} Z+\hbar^{4} U_{0}^{\widehat{\otimes} 3} \subset\left(U_{0}^{\prime}\right)^{\widehat{\otimes} 3}+\hbar^{4} U_{0}^{\widehat{\otimes} 3} .
$$

Let $n \geq 3$. Assume that we have constructed $F_{1}, \ldots, F_{n-1}$, and we now construct $F_{n}$. By assumption, $\Phi_{n-1} \in 1^{\otimes 3}+\hbar U_{0}^{\widehat{\otimes} 3}$ is such that

$$
\varphi_{n-1}:=\hbar \log \Phi_{n-1} \in\left(U_{0}^{\prime}\right)^{\widehat{\otimes} 3}+\hbar^{n+1} U_{0}^{\widehat{\otimes} 3} .
$$

Lemma A.2. The quotient $\left(U^{\prime}+\hbar^{n} U\right) /\left(U^{\prime}+\hbar^{n+1} U\right)$ identifies with $U(\mathfrak{g}) / U(\mathfrak{g})_{\leq n}$. In the same way, the quotient $\left(U_{0}^{\prime \widehat{\otimes} k}+\hbar^{n} U_{0}^{\widehat{\otimes} k}\right) /\left(U_{0}^{\prime \widehat{\otimes} k}+\hbar^{n+1} U_{0}^{\widehat{\otimes} k}\right)$ identifies with $U(\mathfrak{g})_{0}^{\otimes k} /\left(U(\mathfrak{g})_{0}^{\otimes k}\right)_{\leq n}$ and the quotient of $\mathfrak{g}$-invariant subspaces

$$
\left(U_{0}^{\prime \widehat{\otimes} k}+\hbar^{n} U_{0}^{\widehat{\otimes} k}\right)^{\mathfrak{g}} /\left(U_{0}^{\prime \widehat{\otimes} k}+\hbar^{n+1} U_{0}^{\widehat{\otimes} k}\right)^{\mathfrak{g}}
$$


identifies with $\left(U(\mathfrak{g})_{0}^{\otimes k}\right)^{\mathfrak{g}} /\left(U(\mathfrak{g})_{0}^{\otimes k}\right)_{\leq n}^{\mathfrak{g}}$.

The inverse of the first isomorphism takes the class of $\beta \in U(\mathfrak{g})$ to the class of $\hbar^{n} \beta \in U^{\prime}+\hbar^{n} U$. Let $\bar{\alpha} \in\left(U(\mathfrak{g})_{0}^{\otimes 3}\right)^{\mathfrak{g}} /\left(\left(U(\mathfrak{g})_{0}^{\otimes 3}\right)_{\leq n+1}\right)^{\mathfrak{g}}$ be the image of the class of $\varphi_{n-1}$ under the isomorphism above. Let $\alpha \in\left(U(\mathfrak{g})_{0}^{\otimes 3}\right)^{\mathfrak{g}}$ be a representative of $\bar{\alpha}$. Then we have $\varphi_{n-1}=\varphi+\hbar^{n+1} \alpha$, where

$$
\varphi \in\left(U_{0}^{\prime}\right)^{\widehat{\otimes} 3}+\hbar^{n+2} U_{0}^{\widehat{\otimes} 3} .
$$

Now $\varphi_{n-1}$ satisfies the pentagon equation, so

$$
\begin{aligned}
&\left(-\varphi-\hbar^{n+1} \alpha\right)^{1,2,34} \star_{\hbar}\left(\varphi+\hbar^{n+1} \alpha\right)^{2,3,4} \star_{\hbar}\left(\varphi+\hbar^{n+1} \alpha\right)^{1,23,4} \\
& \star_{\hbar}\left(\varphi+\hbar^{n+1} \alpha\right)^{1,2,3} \star_{\hbar}\left(-\varphi-\hbar^{n+1} \alpha\right)^{12,3,4}=0,
\end{aligned}
$$

where $a \star_{\hbar} b$ is the $\mathrm{CBH}$ product for the Lie bracket $[a, b]_{\hbar}=[a, b] / \hbar$.

Lemma A.3. Assume that $n \geq 2$. If $f_{1}, f_{2} \in\left(U_{0}^{\prime}\right)^{2}+\hbar^{n+1} U_{0}$ and $g, h \in \hbar^{n} U_{0}$, then $\left(f_{1}+g\right) \star_{\hbar}\left(f_{2}+h\right)=g+h$ modulo $\left(U_{0}^{\prime}\right)^{2}+\hbar^{n+1} U_{0}$.

Proof. The contribution of the degree 1 part of the $\mathrm{CBH}$ series is $f_{1}+g+f_{2}+h$, which gives $g+h$ modulo $\left(U_{0}^{\prime}\right)^{2}+\hbar^{n+1} U_{0}$.

We now prove that $\left[\left(U_{0}^{\prime}\right)^{2}+\hbar^{n} U_{0},\left(U_{0}^{\prime}\right)^{2}+\hbar^{n} U_{0}\right]_{\hbar} \subset\left(U_{0}^{\prime}\right)^{2}+\hbar^{n+1} U_{0}$. Indeed, we have $\left[U_{0}^{\prime}, U_{0}^{\prime}\right]_{\hbar} \subset U_{0}^{\prime}$, hence $\left[\left(U_{0}^{\prime}\right)^{2},\left(U_{0}^{\prime}\right)^{2}\right]_{\hbar} \subset\left(U_{0}^{\prime}\right)^{2} ;\left[\hbar^{n} U_{0}, \hbar^{n} U_{0}\right]_{\hbar} \subset \hbar^{2 n-1} U_{0} \subset$ $\hbar^{n+1} U_{0}$; and $\left[U_{0}^{\prime}, \hbar^{n} U_{0}\right]_{\hbar} \subset \hbar^{n} U_{0}$, since $U_{0}^{\prime} \subset \hbar U_{0}$, so that

$$
\left[\left(U_{0}^{\prime}\right)^{2}, \hbar^{n} U_{0}\right]_{\hbar} \subset \hbar^{n}\left(U_{0} U_{0}^{\prime}+U_{0}^{\prime} U_{0}\right) \subset \hbar^{n+1}\left(U_{0}\right)^{2},
$$

again because $U_{0}^{\prime} \subset \hbar U_{0}$.

It follows that the contributions of all the higher degree parts of the $\mathrm{CBH}$ series belong to $\left(U_{0}^{\prime}\right)^{2}+\hbar^{n+1} U_{0}$. This implies the lemma.

We continue the proof of the proposition. Lemma A.3 implies that the image of $(\mathrm{A}-2)$ in $\left(U^{\prime \widehat{\otimes} 4}+\hbar^{n+1} U^{\widehat{\otimes} 4}\right) /\left(U^{\prime \widehat{\otimes} 4}+\hbar^{n+2} U^{\widehat{\otimes} 4}\right)=U(\mathfrak{g})^{\otimes 4} /\left(U(\mathfrak{g})^{\otimes 4}\right)_{\leq n+2}$ gives $d(\bar{\alpha})=0$, where

$$
d: U(\mathfrak{g})^{\otimes 3} /\left(U(\mathfrak{g})^{\otimes 3}\right)_{\leq n+2} \rightarrow U(\mathfrak{g})^{\otimes 4} /\left(U(\mathfrak{g})^{\otimes 4}\right)_{\leq n+2}
$$

is the map induced by the Cartier differential.

According to [Drinfeld 1989b], the cohomology of the complex $C^{2} \rightarrow C^{3} \rightarrow C^{4}$ vanishes, where $C^{k}=\left(U(\mathfrak{g})_{0}^{\otimes k}\right)^{\mathfrak{g}} /\left(U(\mathfrak{g})_{0}^{\otimes k}\right)_{\leq n+2}^{\mathfrak{g}}$.

It follows that there exists $\bar{\beta} \in C^{2}$, such that $\bar{\alpha}=d(\bar{\beta})$. Let $\beta \in\left(U(\mathfrak{g})_{0}^{\otimes 2}\right)^{\mathfrak{g}}$ be a representative of $\bar{\beta}$. Set $F_{n}:=\exp \left(\hbar^{n} \beta\right)$ and $\Phi_{n}={ }^{F_{n}} \Phi_{n-1}$.

We get

$$
\varphi_{n}=f_{n}^{2,3} \star_{\hbar} f_{n}^{1,23} \star_{\hbar} \varphi_{n-1} \star_{\hbar}\left(-f_{n}^{12,3}\right) \star_{\hbar}\left(-f_{n}^{1,2}\right),
$$


where $f_{n}=\hbar^{n+1} \beta$. According to Lemma A.3, the class of $\varphi_{n}$ in

$$
\left(\left(U_{0}^{\prime}\right)^{\widehat{\otimes} 3}+\hbar^{n+1} U_{0}^{\widehat{\otimes} 3}\right) /\left(\left(U_{0}^{\prime}\right)^{\widehat{\otimes} 3}+\hbar^{n+2} U_{0}^{\widehat{\otimes} 3}\right)
$$

is $\bar{\alpha}-d(\bar{\beta})=0$, hence $\varphi_{n} \in\left(U_{0}^{\prime}\right)^{\widehat{\otimes} 3}+\hbar^{n+2} U_{0}^{\widehat{\otimes} 3}$. This proves the induction step.

\section{Acknowledgment}

We thank V. Dolgushev, P. Etingof and L.-C. Li for discussions.

\section{References}

[Alekseev 1997] A. Y. Alekseev, "On Poisson actions of compact Lie groups on symplectic manifolds”, J. Differential Geom. 45:2 (1997), 241-256. MR 99b:58086 Zbl 0912.53018

[Boalch 2001] P. P. Boalch, "Stokes matrices, Poisson Lie groups and Frobenius manifolds", Invent. Math. 146:3 (2001), 479-506. MR 2003a:53127 Zbl 1044.53060

[Chloup-Arnould 1997] V. Chloup-Arnould, "Linearization of some Poisson-Lie tensor", J. Geom. Phys. 24:1 (1997), 46-52. MR 99a:58062 Zbl 0888.22006

[Drinfeld 1987] V. G. Drinfeld, “Quantum groups”, pp. 798-820 in Proceedings of the International Congress of Mathematicians (Berkeley, 1986), vol. 1, edited by A. M. Gleason, Amer. Math. Soc., Providence, RI, 1987. MR 89f:17017 Zbl 0667.16003

[Drinfeld 1989a] V. G. Drinfeld, "Almost cocommutative Hopf algebras", Algebra i Analiz 1:2 (1989), 30-46. In Russian; translation in Leningrad Math. J. 1:2, (1990), 321-342. MR 91b:16046 Zbl 0718.16035

[Drinfeld 1989b] V. G. Drinfeld, "Quasi-Hopf algebras”, Algebra i Analiz 1:6 (1989), 114-148. In Russian; translation in Leningrad Math. J. 1:6, (1990), 1419-1457. MR 91b:17016 Zbl 0718.16033

[Duflo 1969] M. Duflo, "Caractères des algèbres de Lie résolubles", C. R. Acad. Sci. Paris Sér. A-B 269 (1969), A437-A438. MR 40 \#4320 Zbl 0254.17006

[Enriquez and Halbout 2003] B. Enriquez and G. Halbout, "An $\hbar$-adic valuation property of universal R-matrices", J. Algebra 261:2 (2003), 434-447. MR 2004a:17015 Zbl 1015.17008

[Enriquez and Halbout 2004] B. Enriquez and G. Halbout, "Poisson algebras associated to quasiHopf algebras", Adv. Math. 186:2 (2004), 363-395. MR 2005c:17029 Zbl 1061.17016

[Enriquez et al. 2003] B. Enriquez, F. Gavarini, and G. Halbout, "Uniqueness of braidings of quasitriangular Lie bialgebras and lifts of classical $r$-matrices", Int. Math. Res. Not. 46 (2003), 24612486. MR 2004m:17045 Zbl 1044.17019

[Enriquez et al. 2005] B. Enriquez, P. Etingof, and I. Marshall, "Comparison of Poisson structures and Poisson-Lie dynamical $r$-matrices", Int. Math. Res. Not. 36 (2005), 2183-2198. MR 2006f: 53120 Zbl 1092.53057

[Etingof and Kazhdan 1996] P. Etingof and D. Kazhdan, "Quantization of Lie bialgebras. I", Selecta Math. (N.S.) 2:1 (1996), 1-41. MR 97f:17014 Zbl 0863.17008

[Gavarini 2002] F. Gavarini, "The quantum duality principle”, Ann. Inst. Fourier (Grenoble) 52:3 (2002), 809-834. MR 2003d:17016 Zbl 1054.17011

[Ginzburg and Weinstein 1992] V. L. Ginzburg and A. Weinstein, "Lie-Poisson structure on some Poisson Lie groups”, J. Amer. Math. Soc. 5:2 (1992), 445-453. MR 92h:17022 Zbl 0766.58018 
[Semenov-Tian-Shansky 1984] M. A. Semenov-Tyan-Shansky, "Classical $r$-matrices and quantization”, Zap. Nauchn. Sem. Leningrad. Otdel. Mat. Inst. Steklov. 133 (1984), 228-235. In Russian; translation in J. Soviet Math. 31:6, (1985), 3411-3416. MR 85m:58083 Zbl 0538.58015

[Semenov-Tian-Shansky 1985] M. A. Semenov-Tian-Shansky, "Dressing transformations and Poisson group actions", Publ. Res. Inst. Math. Sci. 21:6 (1985), 1237-1260. MR 88b:58057 Zbl 0674. 58038

Received April 4, 2005. Revised November 23, 2005.

\author{
BENJAMIN ENRIQUEZ \\ IRMA (CNRS) \\ DÉPARTEMENT DE MATHÉMATIQUES \\ UNIVERSITÉ LOUIS PASTEUR \\ 7, Rue René DESCARTES \\ F-67 084 STRASBOURG \\ FRANCE \\ enriquez@math.u-strasbg.fr \\ Gilles Halbout \\ IRMA (CNRS) \\ DÉPARTEMENT DE MATHÉMATIQUES \\ UNIVERSITÉ LOUIS PASTEUR \\ 7, Rue René Descartes \\ F-67084 STRASBOURG \\ FRANCE \\ halbout@math.u-strasbg.fr
}

